\title{
ddRADseq-assisted construction of a high- density SNP genetic map and QTL fine mapping for growth-related traits in the spotted scat (Scatophagus argus)
}

Wei Yang ${ }^{1,2}$, Yaorong Wang ${ }^{1}$, Dongneng Jiang ${ }^{1}$, Changxu Tian ${ }^{1}$, Chunhua Zhu' ${ }^{1}$ Guangli Li ${ }^{1}$ and Huapu Chen ${ }^{1 *}$

\begin{abstract}
Background: Scatophagus argus is a popular farmed fish in several countries of Southeast Asia, including China. Although S. argus has a highly promising economic value, a significant lag of breeding research severely obstructs the sustainable development of aquaculture industry. As one of the most important economic traits, growth traits are controlled by multiple gene loci called quantitative trait loci (QTLS). It is urgently needed to launch a marker assisted selection (MAS) breeding program to improve growth and other pivotal traits. Thus a high-density genetic linkage map is necessary for the fine mapping of QTLs associated with target traits.

Results: Using restriction site-associated DNA sequencing, 6196 single nucleotide polymorphism (SNP) markers were developed from a full-sib mapping population for genetic map construction. A total of 6193 SNPs were grouped into 24 linkage groups (LGs), and the total length reached $2191.65 \mathrm{cM}$ with an average marker interval of $0.35 \mathrm{cM}$. Comparative genome mapping revealed 23 one-to-one and 1 one-to-two syntenic relationships between S. argus LGs and Larimichthys crocea chromosomes. Based on the high-quality linkage map, a total of 44 QTLS associated with growth-related traits were identified on 11 LGs. Of which, 19 significant QTLs for body weight were detected on 9 LGs, explaining 8.8-19.6\% of phenotypic variances. Within genomic regions flanking the SNP markers in QTL intervals, we predicted 15 candidate genes showing potential relationships with growth, such as Hbp 1, Vgll4 and Pim3, which merit further functional exploration.
\end{abstract}

Conclusions: The first SNP genetic map with a fine resolution of $0.35 \mathrm{cM}$ for $\mathrm{S}$. argus has been developed, which shows a high level of syntenic relationship with $L$. crocea genomes. This map can provide valuable information for future genetic, genomic and evolutionary studies. The QTLs and SNP markers significantly associated with growthrelated traits will act as useful tools in gene mapping, map-based cloning and MAS breeding to speed up the genetic improvement in important traits of $S$. argus. The interesting candidate genes are promising for further investigations and have the potential to provide deeper insights into growth regulation in the future.

Keywords: Scatophagus argus, Linkage mapping, Quantitative trait locus, Comparative genomics, Growth-related genes, RADseq

\footnotetext{
* Correspondence: chpsysu@hotmail.com

'Southern Marine Science and Engineering Guangdong Laboratory

(Zhanjiang), Guangdong Research Center on Reproductive Control and

Breeding Technology of Indigenous Valuable Fish Species, Fisheries College,

Guangdong Ocean University, Zhanjiang 524088, China

Full list of author information is available at the end of the article
}

(c) The Author(s). 2020 Open Access This article is licensed under a Creative Commons Attribution 4.0 International License, which permits use, sharing, adaptation, distribution and reproduction in any medium or format, as long as you give appropriate credit to the original author(s) and the source, provide a link to the Creative Commons licence, and indicate if changes were made. The images or other third party material in this article are included in the article's Creative Commons licence, unless indicated otherwise in a credit line to the material. If material is not included in the article's Creative Commons licence and your intended use is not permitted by statutory regulation or exceeds the permitted use, you will need to obtain permission directly from the copyright holder. To view a copy of this licence, visit http://creativecommons.org/licenses/by/4.0/. The Creative Commons Public Domain Dedication waiver (http://creativecommons.org/publicdomain/zero/1.0/) applies to the data made available in this article, unless otherwise stated in a credit line to the data. 


\section{Introduction}

High-quality fish breed (strain) is the primary prerequisite for large-scale commercial culture. Successful aquaculture largely depends on genetic breeding for rapider growth rate, larger size, higher survival rate, better eating quality, and so on [1]. In many cultured economic aquatic animals, substantial improvement has been achieved using conventional selective breeding approaches. However, economically important traits such as growth, disease resistance, temperature tolerance and flesh quality are mostly governed by quantitative trait loci (QTLs), which are defined as chromosomal regions involving single genes or gene clusters [2]. For genetic improvement of quantitative traits, conventional breeding strategies such as family and individual selection mainly rely on the phenotype and pedigree information [3], whereas the underlying genes showing minor effects usually bring in unwanted nondeterminacy. With the big advance and increasing application of modern biotechnology, marker-assisted selection (MAS) and genomic selection using markers linked to QTLs are more effective in accelerating the genetic breeding process by improving the accuracy of selection and by speeding up genetic improvement through direct and early selection $[3,4]$. As a fertile area of research on genetic breeding, QTL mapping based on genotypic data has become an important technique to facilitate the investigations on quantitative traits, and can lay an effective way to understand potential location information and numbers of linked markers for beneficial target traits [5].

A genetic linkage map is a helpful tool possessing tremendous potential to facilitate QTL mapping for target traits with economic values as well as genomics and genetics studies, including map-based cloning, comparative genome analysis, and whole-genome assembly [3]. For aquaculture fishes, two genetic linkage maps were first constructed in Oncorhynchus mykiss [6] and Oreochromis niloticus [7] around the same time. And during the past 20 years, genetic breeding experts have constructed numbers of genetic maps utilizing various types of molecular markers, such as AFLP (amplified fragment length polymorphism), RAPD (random amplified polymorphic DNA) and SSR (simple sequence repeat), in many kinds of aquaculture animals, including over 30 fish species. However, a majority of existing linkage maps have low marker density, their abilities to assist in the fine-scale mapping of QTLs and other studies were seriously limited. Compared with other types of marker, single nucleotide polymorphisms (SNPs) can be genotyped on a more abundant and much larger scale [8], which has become the most popular type of codominant marker for the construction of genetic maps with higher marker-density and resolution. Due to the high cost and laborious work for SNP genotyping, however, there was a big challenge to obtain a large number of SNPs and genotype in relative large mapping families [9]. Benefiting from the rapid development of next-generation sequencing (NGS) technology in the past decade, varieties of genotyping-by-sequencing (GBS) techniques have been created and widely employed in time-saving and cost-effective SNP markers discovery and genotyping throughout the genome, even in non-model species [10]. Of these methods, restriction site-associated DNA sequencing (RADseq) and its derivative methods ddRAD [11], SLAF [12] and 2b-RAD [13] have been successfully utilized for high density (HD) linkage maps construction in many fish species, such as Scophthalmus maximus [14], Salmo salar [15], Paralichthys olivaceus [16], O. niloticus [17], and Lates calcarifer [18].

As one of the most important quantitative traits controlled by multi-gene QTLs as well as environmental factors, fish growth can directly affect the yield of aquaculture [19]. Delightedly, QTL mapping enables us not only to detect genetic markers associated with the genetic variation for important traits but also to find out the candidate genes involving in the regulatory processes of target traits [9]. Up to now growth-related traits have been mapped and well-studied in a wide variety of fish species with economic importance. Significant QTLs associated with growth traits have been identified, and in most cases growth-related QTLs are distributed on multiple linkage groups, e.g., 14 QTLs on 8 LGs in Yellow River carp [1], 21 QTLs on 12 LGs in Yangtze River common carp [3], 28 QTLs on 5 LGs in Pseudobagrus ussuriensis [9], 6 QTLs on 6 LGs in L. calcarifer [18], and 23 QTLs on 4 LGs in Trachinotus blochii [20]. These research findings have been greatly accelerating the progress of genetic improvement in economic fishes via providing powerful tools for MAS breeding.

The spotted scat Scatophagus argus (order Perciformes, family Scatophagidae) generally inhabits around the Indo-Pacific region, including southeast China [21]. Owing to its notable features such as high nutritional value, easy cultivation, low feeding cost and strong disease resistance, $S$. argus has become a popular aquaculture fish species in southeast Asia [22]. According to an incomplete survey, it has become a valuable species presently and been widely cultured in Guangdong, Guangxi, and Taiwan provinces of China with an annual output value of approximately RMB 150 Million. The commercial demand for seedlings has constantly grown over recent years. In view of its economic importance, $S$. argus has been intensively studied on reproductive biology, especially on artificial inducing [23-27] and mechanism of reproductive regulation [28-33] in recent years. Artificial propagation studies have been carried out since the year 2003 in China [34] and fortunately, a highly efficient technique had been established several years ago [25, 35]. However, few genetic and genomic 
Table 1 Pearson's correlation coefficients for all pairwise combinations of the eight growth-related traits of spotted scat F1 full-sib family $(P<0.001$ for all)

\begin{tabular}{lllllllll}
\hline Traits & BW & TL & BL & BH & PD & PA & HL & CPH \\
\hline BW & 1 & 0.864 & 0.865 & 0.872 & 0.627 & 0.789 & 0.634 & 0.817 \\
TL & & 1 & 0.993 & 0.902 & 0.625 & 0.831 & 0.644 & 0.836 \\
BL & & & 1 & 0.906 & 0.624 & 0.841 & 0.662 & 0.832 \\
BH & & & & 1 & 0.540 & 0.786 & 0.614 & 0.811 \\
PD & & & & & 1 & 0.703 & 0.708 & 0.630 \\
PA & & & & & & 1 & 0.742 & 0.859 \\
HL & & & & & & 1 & 0.661 \\
CPH & & & & & & & 1
\end{tabular}

$B W$ body weight, $T L$ total length, $B L$ body length, $B H$ body height, $P D$ predorsal length, $P A$ pre-anal length, $H L$ head length, $C P H$ caudal peduncle height.

studies have been reported for $S$. argus yet. As with many other farmed fish, the serious lag of breeding research and declining population resource have resulted in certain regressions in growth traits and disease resistance of cultured fish, which can seriously impact the quality and safety of food fish products. Hence it is urgent to launch a breeding program to promote the sustainable development of $S$. argus fish industry by improving important genetic traits.

In this study, we applied double digest restriction siteassociated DNA sequencing (ddRADseq) method to identify thousands of high-quality polymorphic SNP markers by genotyping a full-sib mapping family of $S$. argus. Then linkage mapping and QTL analysis were performed. The main purposes of our study were to obtain a HD SNP-based genetic map, identify a number of growth-related QTLs with large effects and significant markers for possible use in MAS, and to provide potential genes for further studies on regulatory mechanism of growth.

\section{Results}

Phenotypic analysis of growth-related traits

Eight growth-related traits of the mapping family consisting of 420 full-sib progeny were measured and investigated. Kolmogorov-Smirnov tests were performed and the results indicated that these measured traits were totally in concordance with normal distribution $(P>0.05)$. The phenotypic variations and frequency distribution of these growth traits are shown in Additional file 1: Table S1 and Additional file 2: Figure S1. The mean values \pm $\mathrm{SD}$ of BW, TL, BL, BH, HL, PD, PA and $\mathrm{CPH}$ were $81.906 \pm 17.751 \mathrm{~g}, \quad 14.605 \pm 0.955 \mathrm{~cm}, 12.399 \pm 0.849 \mathrm{~cm}$, $7.100 \pm 0.552 \mathrm{~cm}, \quad 3.051 \pm 0.251 \mathrm{~cm}, \quad 3.723 \pm 0.342 \mathrm{~cm}$, $8.183 \pm 0.600 \mathrm{~cm}$ and $1.585 \pm 0.120 \mathrm{~cm}$, respectively. Their phenotypic values displayed abundant variations, especially for BW, in which the highest coefficient of variation $(21.67 \%)$ was observed. Pearson's correlation analysis was also conducted and all growth-related traits showed a significant correlation with each other $(r=$ $0.540 \sim 0.993, P<0.001$ ) (Table 1$)$. Specifically, BW significantly correlated with $\mathrm{BH}(r=0.872)$, BL $(r=0.865)$ and TL $(r=0.864)$. The highest correlation coefficient value was observed between $\mathrm{BL}$ and TL $(r=0.993)$, followed by that between $\mathrm{BH}$ and $\mathrm{BL}(r=0.906)$, while the weakest correlation occurred between $\mathrm{PD}$ and $\mathrm{BH}$ $(r=0.540)$.

\section{ddRAD libraries sequencing}

The ddRAD libraries of two parents and their 200 fullsib offspring were sequenced on an Illumina Hiseq2500 platform. A total of $1753,714,542$ raw reads (150 bp in length) were obtained, comprising approximately 257.8 Gb sequencing data (Table 2). The average reads number of the standard libraries for two parents was 59,071, 871 , whereas that of each progeny was $8,177,854$. The sequencing depth of each parent and progeny reached an average of $14.5 \times$ and $2.0 \times$, respectively. Subsequent

Table 2 Statistic summary of the ddRADseq data for the mapping population of spotted scat

\begin{tabular}{lllll}
\hline Type & Item & Female parent & Male parent & Average of progeny \\
\hline Raw data & GC_Rate (\%) & 40.17 & 40.16 & 40.66 \\
& Q20_Rate (\%) & 96.77 & 96.74 & 94.71 \\
& Q30_Rate (\%) & 91.87 & 91.85 & 89.35 \\
& Raw reads & $57,224,366$ & $60,919,376$ & $8,177,854$ \\
& Raw base (bp) & $8,411,981,802$ & $8,955,148,272$ & $1,202,173,359$ \\
Clean data & Depth & $195.10 \times$ & $207.69 \times$ & $27.88 \times$ \\
& GC_Rate (\%) & 40.12 & 40.12 & 40.58 \\
& Q20_Rate (\%) & 96.77 & 96.74 & 94.75 \\
& Q30_Rate (\%) & 91.87 & 91.84 & 89.40 \\
Clean reads & $56,800,034$ & $60,536,986$ & $8,099,577$ \\
Clean base (bp) & $8,345,222,442$ & $8,894,041,121$ & $1,189,864,968$ \\
\end{tabular}


trimming, quality filtering and low-quality reads removing finally generated $1,737,252,420$ clean reads in total. The female and male parental data contained 56,800,034 filtered reads with a Q20_Rate of $96.77 \%$ and 60,536,986 filtered reads with a Q20_Rate of 96.74\%, respectively. An average of 8,099,577 clean reads was produced for each individual of the offspring, which was equivalent to approximate $1.19 \mathrm{~Gb}$ of data.

\section{SNP detection and genotyping}

Based on ddRADseq of the $S$. argus mapping family and bioinformatics analysis, a total of 88,789 original polymorphic markers were detected using the STACKS pipeline. Through stringent screening, 20,921 high-quality polymorphic SNP markers were successfully genotyped in both parents and at least $90 \%$ of the offspring (Additional file 3: Table S2). Of which, 17,663 (84.43\%) parent-specific SNP loci were heterozygous in either of the parents, and 3258 (15.57) SNPs were heterozygous in both of the parents. After segregation distortion tests, 6196 (29.62\%) SNPs that were consistent with a Mendelian segregation pattern $(P \geq 0.01)$ were finally retained and utilized in the following linkage analysis (Table 3). All Mendelian SNPs were classified into three categories based on their segregation types, the marker numbers for maternal heterozygosity $(\mathrm{lm} \times \mathrm{ll})$ and paternal heterozygosity $(\mathrm{nn} \times \mathrm{np})$ were 2566 (41.41\%) and 2683 (43.30\%), respectively; and the remaining 947 (15.28\%) markers were heterozygous in both parents (hk $\times$ hk and ef $\times$ eg).

\section{Construction of genetic linkage maps}

Using the JoinMap 4.1 software with a LOD threshold of 8.0, a consensus genetic map was constructed. A total of 6193 (99.9\%) out of the 6196 polymorphic SNP markers were successfully grouped into 24 linkage groups (LGs), spanning a total length of $2191.65 \mathrm{cM}$ with an average marker interval of $0.35 \mathrm{cM}$ (Table 4 and Fig. 1). The number of LGs is perfectly consistent with the diploid chromosome number of $S$. argus $(2 N=48)$ [36]. The number of mapped markers in each LG varied from 137 (LG4) to 351 (LG20) with an average of 258 SNPs per group. The longest LG was $127.09 \mathrm{cM}$ (LG12) in length and the shortest group was only $59.96 \mathrm{cM}$ (LG4) in

Table 3 Statistic information of Mendelian SNP markers showing heterozygosity in one or both parents

\begin{tabular}{llll}
\hline Segregation patterns & Segregation ratio & Number of SNP loci & Ratio (\%) \\
\hline $\mathrm{hk} \times \mathrm{hk}$ & $1: 2: 1$ & 932 & 15.04 \\
$\mathrm{Im} \times \|$ & $1: 1$ & 2566 & 41.41 \\
$\mathrm{nn} \times \mathrm{np}$ & $1: 1$ & 2683 & 43.30 \\
$\mathrm{ef} \times \mathrm{eg}$ & $1: 1: 1: 1$ & 15 & 0.24 \\
$\mathrm{ab} \times \mathrm{cd}$ & $1: 1: 1: 1$ & 0 & 0 \\
Total & $\backslash$ & 6196 & 1 \\
\hline
\end{tabular}

length, whereas average intervals between two adjacent markers ranged from $0.24 \mathrm{cM}$ (LG15) to $0.58 \mathrm{cM}$ (LG13). Based on two commonly-used estimating methods [37, 38], the expected genome length was estimated to be $2209.20 \mathrm{cM}\left(\mathrm{G}_{\mathrm{e} 1}\right)$ and $2209.31 \mathrm{cM}\left(\mathrm{G}_{\mathrm{e} 2}\right)$, with an average of $2209.25 \mathrm{cM}\left(\mathrm{G}_{\mathrm{e}}\right)$. Herein the genome coverage of this linkage map reached 99.2\% (Additional file 7: Table S4). As the genome size of S. argus has been estimated to be $598.73 \mathrm{Mb}$ (unpublished data), the average recombination rate across all LGs was $\sim 3.7 \mathrm{cM}$ per $\mathrm{Mb}$.

Two sex-specific maps each consisting of 24 LGs were also constructed (Additional file 4: Table S3, Additional file 5: Figure S2 and Additional file 6: Figure S3). The female map spanned a total length of $2290.56 \mathrm{cM}$ with an average inter marker distance of $0.65 \mathrm{cM}$, whereas the male map spanned a total genetic distance of 1880.23 $\mathrm{cM}$ with an average marker interval of $0.52 \mathrm{cM}$. The genetic length of individual LGs of female and male maps varied from $43.12 \mathrm{cM}$ (LG4) to $137.55 \mathrm{cM}$ (LG12) and from $44.49 \mathrm{cM}$ (LG4) to $126.56 \mathrm{cM}$ (LG12), respectively. In order to validate the quality of genetic maps, synteny analyses between the consensus map and female or male map were performed. The syntenic relationships of shared markers between the consensus map and sexspecific maps were highly consistent (Additional file 8: Figure S4).

\section{Comparative genome mapping}

Successful construction of S.argus genetic map provided a framework to compare its conserved genomic regions with those of other teleosts. Homology searches against the genomes of 10 model or non-model fishes were explored using the ddRAD loci mapped in S.argus genetic map (Fig. 2). The fewest number of homologous ddRAD loci were observed in the comparison with $D$. rerio (56), followed by comparisons with I. punetaus (77) and $S$. salar (94). Homologous sequences to more than $400 \mathrm{~S}$. argus ddRAD loci were found in L. crocea (978), O. niloticus (472), and P. olivaceus (463) (Fig. 2a). Moreover, Oxford grids were made for $S$. argus against above three teleosts based on the number of orthologous markers on each LG or chromosome. All 24 pairs of LGs or chromosomes in $S$. argus and $L$. crocea showed a basically clear 1:1 syntenic relationship (Fig. 2b), indicating a relatively high-level of genomic synteny between these two species. Comparisons with the other two fish species also indicated highly conservative 1:1 relationships, although several 1:2 syntenic relationships were observed across the genomic regions (Fig. 2c and d). For example, $O$. niloticus chromosome 7 corresponded to LG1 and LG16 in S. argus (Fig. 2c). P. olivaceus chromosome 23 corresponded to LG7 and LG9 in S. argus (Fig. 2d). Of these fish species analyzed, $L$. crocea exhibited by far the 
Table 4 Summary of the SNP-based high-density genetic map of spotted scat

\begin{tabular}{|c|c|c|c|c|}
\hline Linkage group & Number of markers & Genetic length (cM) & Marker interval (cM) & Maximum gap (cM) \\
\hline LG1 & 316 & 87.78 & 0.28 & 3.20 \\
\hline LG2 & 254 & 91.20 & 0.36 & 5.03 \\
\hline LG3 & 252 & 82.83 & 0.33 & 2.18 \\
\hline LG4 & 137 & 59.96 & 0.44 & 2.56 \\
\hline LG5 & 193 & 60.89 & 0.32 & 1.74 \\
\hline LG6 & 218 & 92.66 & 0.43 & 2.91 \\
\hline LG7 & 285 & 95.06 & 0.33 & 2.12 \\
\hline LG8 & 260 & 92.34 & 0.36 & 5.52 \\
\hline LG9 & 295 & 85.88 & 0.29 & 3.81 \\
\hline LG10 & 276 & 89.44 & 0.32 & 4.38 \\
\hline LG11 & 278 & 95.13 & 0.34 & 2.44 \\
\hline LG12 & 291 & 127.09 & 0.44 & 4.89 \\
\hline LG13 & 202 & 117.17 & 0.58 & 5.71 \\
\hline LG14 & 236 & 88.52 & 0.38 & 2.91 \\
\hline LG15 & 305 & 73.94 & 0.24 & 1.46 \\
\hline LG16 & 232 & 92.51 & 0.40 & 7.27 \\
\hline LG17 & 231 & 101.80 & 0.44 & 5.33 \\
\hline LG18 & 327 & 112.81 & 0.34 & 9.09 \\
\hline LG19 & 216 & 89.17 & 0.41 & 1.76 \\
\hline LG20 & 351 & 105.94 & 0.30 & 2.28 \\
\hline LG21 & 310 & 85.41 & 0.28 & 1.95 \\
\hline LG22 & 238 & 97.44 & 0.41 & 2.57 \\
\hline LG23 & 150 & 78.19 & 0.52 & 5.38 \\
\hline LG24 & 340 & 88.48 & 0.26 & 1.52 \\
\hline Maximum & 351 & 127.09 & 0.58 & 9.09 \\
\hline Minimum & 137 & 59.96 & 0.24 & 1.46 \\
\hline Total & 6193 & 2191.65 & 8.79 & 87.99 \\
\hline Average & 258 & 91.32 & 0.35 & 3.67 \\
\hline
\end{tabular}

closest phylogenetic relationship with $S$. argus. L. crocea chromosomes appear to show a high degree of syntenic relationship with the $S$. argus LGs, as every chromosome is clearly linked to one linkage group in $S$. argus with an exception of chromosome 8 (Fig. 2b). Of the 956 markers uniquely anchored to $L$. crocea chromosomes, $832(87.0 \%)$ were located into syntenic boxes (Fig. 2e). On the whole, there is a strong correlation of each $S$. argus linkage group to a single chromosome not only in L. crocea but also in O. niloticus, which are both belong to Perciformes. Our investigation primarily validated the reliability of $S$. argus linkage map, which will establish informative genome resources for future studies.

\section{QTL analysis for growth traits}

According to the Pearson's correlation coefficients, five growth traits (BW, TL, BL, $\mathrm{BH}$ and $\mathrm{CPH}$ ) showing relatively high correlation with each other were selected for
QTL analysis in this study. As determined by permutation tests, the estimated values of chromosome-wide $(\mathrm{CW})$ and genome-wide (GW) significance thresholds for growth-related traits varied from 3.4 to 3.8 and 5.3 to 5.4 , respectively. By using MQM method in MapQTL 5.0, a total of 44 QTLs associated with growth traits were detected on $11 \mathrm{LGs}$, including $17 \mathrm{GW}$ significant QTLs and $27 \mathrm{CW}$ significant QTLs with LOD scores ranging from 4.21 to 7.88 (Table 5 and Fig. 3). LG24 had the highest number of QTLs (11), followed by LG2 (10) and LG7 (8), while LG8, LG9, LG13 and LG15 each only contained one. A total of 19 QTLs associated with body weight were distributed on 9 LGs (LG2, LG5, LG7, LG11, LG13, LG15, LG19, LG21 and LG24) with phenotypic variance explained (PVE) values ranging from $8.8 \%$ (qBW15-1) to $19.6 \%$ (qBW2-1). Meanwhile, 14 significant QTLs for body height were detected on 7 LGs (LG2, LG5, LG7, LG8, LG9, LG19 and LG24) with PVE 


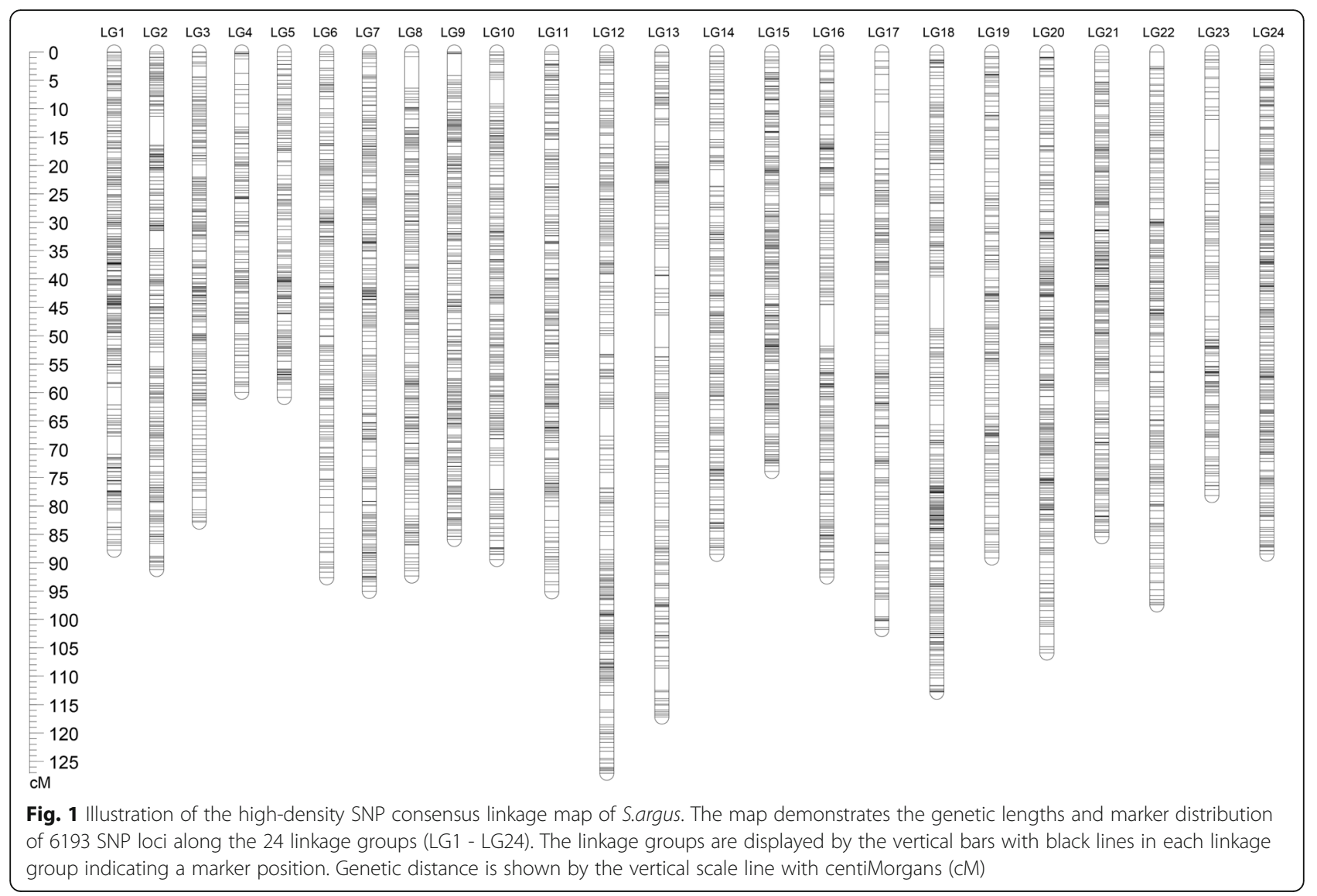

values varying from 9.7\% (qBH8-1) to $16.5 \%$ (qBH2-1). Five QTLs associated with $\mathrm{CPH}(\mathrm{qCPH} 2-1, \mathrm{qCPH} 2-2$, qCPH7-1, qCPH7-2 and qCPH7-3) were located at $37.607 \mathrm{cM}, \quad 58.917 \mathrm{cM}$ along LG2, and $37.527 \mathrm{cM}$, $45.350 \mathrm{cM}, 94.126 \mathrm{cM}$ along LG7, accounting for 18.5 , $13.3,11.1,10.7$ and $11.0 \%$ of the phenotypic variations, respectively. Interestingly, the QTL with relatively higher PVE values were all located at intervals on LG2, e.g., 30.983-40.473 cM for BW, 30.708-37.607 cM for CPH, and $36.998-38.312 \mathrm{cM}$ for $\mathrm{TL}, \mathrm{BL}$ and $\mathrm{BH}$, suggesting that LG2 may play a more important role in growth regulation in S. argus. Specifically, the peak LOD values of QTLs associated with BW, TL, BL, BH and $\mathrm{CPH}$ were located at $37.607 \mathrm{cM}$ of LG2 near the SNP marker R1 98424, contributing to $19.6,16.4,16.9,16.5$, and $18.5 \%$ of the phenotypic variation, respectively (Table 5 ). Because of the high correlation value $(r=0.993)$ between BL and TL, most QTLs for these two traits were located at the overlapped confidence intervals along LG2.

QTLs associated with quantitative traits are generally not randomly distributed across chromosomal regions and chromosomes. Previous investigations had identified a set of QTLs in QTL clusters, which were defined by the presence of multiple QTLs associated with different or similar traits, respectively [39, 40]. In this study, a total of 10 QTL clusters were detected in LG2, LG7, LG19 and LG24 (Fig. 4 and Additional file 9: Table S5). We also noted that the QTLs located in certain clusters were associated with more than three growth traits, e.g., LG2-cluster-1 (30.708-40.473 cM) possessed six QTLs pertaining to all of the five growth traits; LG2-cluster-2 (58.402-59.763 cM) harbored four QTLs related to four growth traits (BW, TL, BL and $\mathrm{CPH}$ ); LG7-cluster-1 (36.461-53.388 cM) possessed four QTLs significantly associated with three growth traits (BW, $\mathrm{BH}$ and $\mathrm{CPH})$. Moreover, the analysis results indicated that QTL confidence intervals in LG2-cluster-2 or LG7-cluster-2, or LG24-cluster-3 displayed a high degree of overlapping with each other. Therefore we can make use of the overlapping regions to further analyze the gene annotation for obtaining more useful information.

\section{Candidate genes for growth}

A total of 24 SNP markers located in the confidence intervals of body weight QTLs were selected and utilized to identify candidate growth-related genes. By means of searching against the $S$. argus genome using ddRAD-Tag sequences, the $50 \mathrm{~kb}$ regions flanking to each SNP marker were obtained from corresponding scaffolds. Based on the annotation information of genome, a total of 32 genes were 


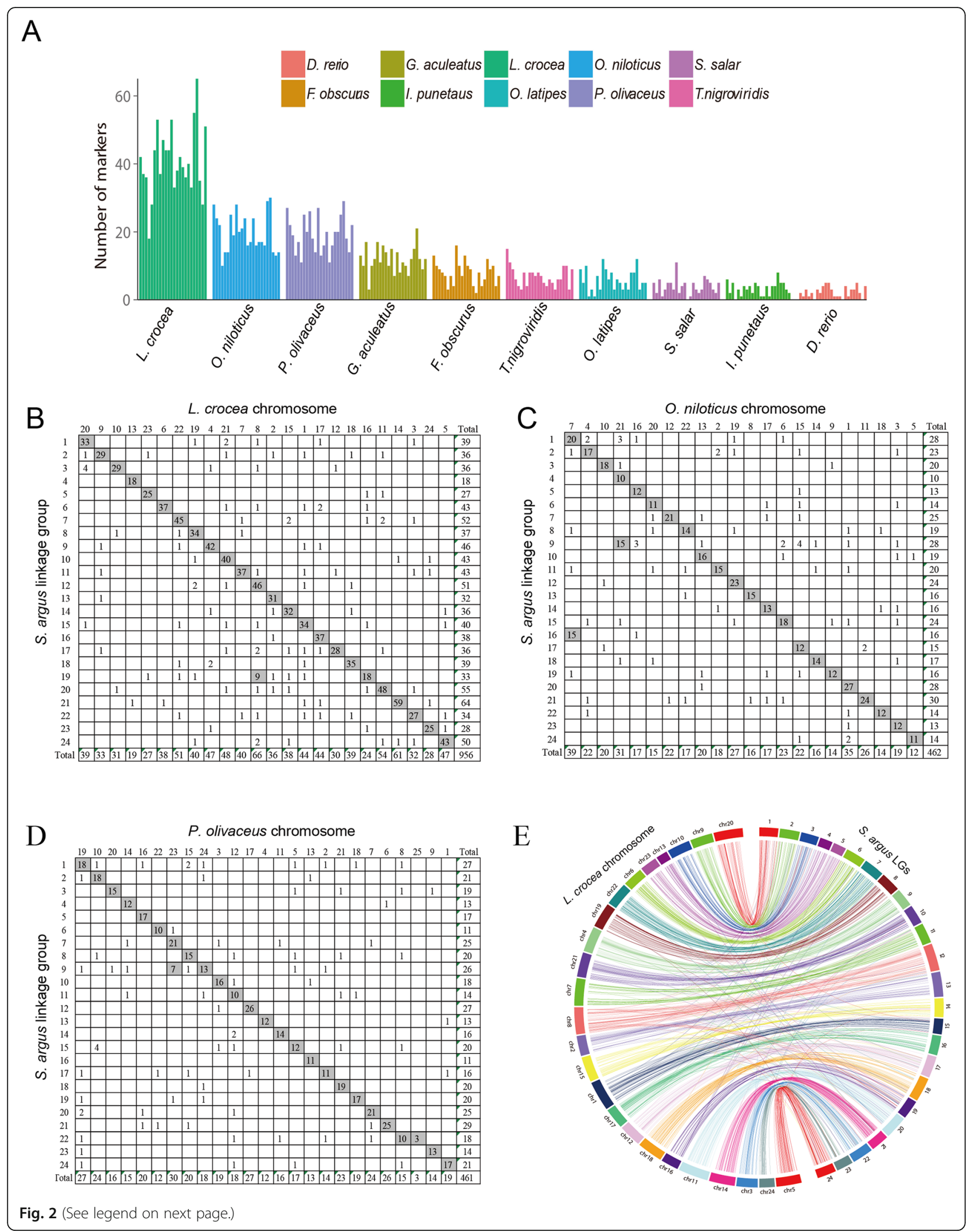


(See figure on previous page.)

Fig. 2 Comparative genomics analysis for the genetic map of S.argus. (a) the numbers of markers per linkage group that showed homology with genome sequences of other fish, listed on the X-axis; (b)-(d) Oxford grids showing conservation of macrosynteny relationships between S.argus linkage groups and chromosomes of L. crocea (b), O. niloticus (c), and P. olivaceus (d). The numbers of S.argus ddRAD markers with significant BLASTn hits in the other fish are presented in box, and the putative syntenic pairs are indicated by gray boxes along the diagonal. (e) Circos diagram representing syntenic relationships between proposed linkage groups of S.argus (right) and published chromosomes of L. crocea (left). only markers on each linkage group of S.argus that were mapped to a single chromosome of L. crocea were illustrated. Each colored arc represents a marker match between linkage group and chromosome

located in these regions (Table 6). Among them, 15 potential growth-related genes were obtained, such as HMG box-containing protein $1(\mathrm{Hbp} 1)$, centrosomal protein of $131 \mathrm{kDa}$ (Cep131), nucleosome assembly protein 1-like 1 (Nap1l1), Ataxin-1 (Atxn1), serine/threonine-protein kinase pim-3 (Pim3), scm-like with four MBT domains protein 1 (Sfmbt1), prickle-like protein 2 (Prickle2), voltagedependent calcium channel subunit alpha-2/delta-3 (Cacna2d3), transcription cofactor vestigial-like protein 4 (Vgll4), apoptotic protease-activating factor 1 (Apaf1), and PDZ domain-containing protein $4(P d z d 2)$, which have been reported to play important parts in cell proliferation and growth, tumorigenesis, tumor suppressor, and muscle regeneration, respectively [41-54]. These candidate genes are worthy of further studies to reveal their exact roles in genetic control of growth traits in the future.

\section{Discussion}

\section{ddRADseq and SNPs genotyping}

As a keystone technology with ability to efficiently and accurately determine genotypes, RADseq and its derivative methods have brought in a revolution for the largescale development of polymorphic SNPs, an excellent tool for population genomics and genetics studies $[3,11$, $55]$. With the increase of sequencing depth and cost reduction of NGS, RADseq has now been widely applied in construction of linkage maps and QTL fine mapping of genetic traits for commercial fishes. For example, genetic map and growth-related QTLs in turbot (6647 SNPs) [56], blunt snout bream (14,648 SNPs) [57], Yangtze River common carp (7820 SNPs and 295 SSRs) [3], golden pompano (12,358 SNPs) [20], Yellow River common carp (6230 SNPs and 65 SSRs) [1], and P. ussuriensis (7435 SNPs) [9]; genetic map and sex determination QTLs in hāpuku (1575 SNPs) [58], mandarin fish (3283 SNPs) [59], channel catfish (4768 SNPs) [60]; linkage map and disease resistance-related QTLs in Asian seabass (6425 SNPs) [61], Japanese flounder (12,712 SNPs) [16]; genetic map and fatty acid compositions-related QTLs in Asian Seabass (2424 SNPs) [62]. Accumulating achievement has reinforced the consensus among geneticist about the advantage of RADseq technology. In the present study for S. argus, ddRADseq-based SNPs discovery in a F1 full-sib family was performed for the first time. A total of $1753.7 \mathrm{M}$ pair-end reads and 293,309 original RAD markers were generated, including 88,789 polymorphic SNP loci with a polymorphism ratio of $30.27 \%$, which was higher than that found in common carp (21.1\%) [12] and pikeperch (14.63\%) [63]. According to our preliminary population genetic analysis (unpublished data), we have inferred that the higher polymorphism rate is due to a high genetic diversity of $S$. argus. Both the sufficient sequencing depths for each progeny and the genotype integrity of these SNP markers (>90\%) in the mapping population guaranteed a high-level of genotyping accuracy (Table 2) and finally, abundant high-quality Mendelian SNPs were retained as effective marker resource for linkage mapping. Our results once again demonstrated that ddRADseq is an effective approach for SNPs identification in linkage mapping researches.

\section{High-quality genetic map}

A genetic linkage map with good quality is a basic genetic tool for fine QTL mapping, map-based gene cloning, and marker-assisted breeding [64]. This study reports the first genetic linkage map of S. argus covering 99.2\% of genome, 6193 SNPs were assigned to 24 LGs (Fig. 1). A diploid chromosome number of $2 N=48$ was identified in $S$. argus by karyotype analysis [36], this result is in agreement with the number of LGs in this map, indicating that each LG corresponds to one chromosome and all $S$. argus chromosomes are covered by this genetic map. Nevertheless, the detailed correspondences require to be clarified in future exploration. Moreover, the female and male linkage maps were constructed. The results of synteny analysis showed that both female and male maps showed a high degree of syntenic relationship with the consensus map (Additional file 8: Figure S4), reflecting a high quality and reliability of the $S$. argus linkage maps. The average marker number of 258 per group and inter-marker distance of $0.35 \mathrm{cM}$ suggested that the consensus map can provide a reference to help position sequence scaffolds on the physical map, which would be beneficial for identifying genes near each SNP locus.

A number of HD genetic maps have already been developed in aquaculture fishes. Compared with most of these linkage maps, the final $S$. argus genetic map containing thousands of SNPs has an improved number of markers 
Table 5 Summary of the results of linkage mapping for QTLS associated with growth traits

\begin{tabular}{|c|c|c|c|c|c|c|c|c|}
\hline Traits & QTL & LG & $\mathrm{Cl}(\mathrm{cM})$ & Nearest marker & Position (cM) & LOD & PVE (\%) & Significance level \\
\hline \multirow[t]{19}{*}{$\overline{B W}$} & qBW2-1 & 2 & $30.983-40.473$ & R1_98424 & 37.607 & 7.76 & 19.6 & GW \\
\hline & qBW2-2 & 2 & $58.402-59.763$ & R1_192243 & 58.917 & 4.61 & 10.1 & $C W$ \\
\hline & qBW5-1 & 5 & $25.992-27.809$ & R1_269416 & 25.992 & 4.83 & 11.2 & $C W$ \\
\hline & qBW7-1 & 7 & $36.461-39.134$ & R1_85816 & 37.461 & 4.77 & 10.9 & CW \\
\hline & qBW7-2 & 7 & $91.849-95.060$ & R1_211226 & 93.370 & 5.85 & 13.4 & GW \\
\hline & qBW11-1 & 11 & $43.640-45.338$ & R1_205729 & 43.64 & 4.35 & 9.6 & CW \\
\hline & qBW11-2 & 11 & $71.138-76.128$ & R1_121023 & 74.822 & 4.38 & 9.6 & CW \\
\hline & qBW13-1 & 13 & $0-0.804$ & R1_113228 & 0.543 & 4.87 & 10.7 & CW \\
\hline & qBW15-1 & 15 & $2.747-5.174$ & R1_162345 & 3.972 & 4.42 & 8.8 & $C W$ \\
\hline & qBW19-1 & 19 & $30.002-30.806$ & R1_140416 & 30.237 & 5.52 & 12.8 & GW \\
\hline & qBW19-2 & 19 & $63.688-67.454$ & R1_198192 & 66.225 & 4.48 & 10.2 & $C W$ \\
\hline & qBW21-1 & 21 & $37.238-41.709$ & R1_68531 & 37.567 & 4.84 & 10.6 & $C W$ \\
\hline & qBW21-2 & 21 & $50.546-53.101$ & R1_71627 & 52.125 & 4.81 & 11.2 & $C W$ \\
\hline & qBW24-1 & 24 & 13.099-19.843 & R1_255417 & 18.653 & 6.25 & 13.7 & GW \\
\hline & qBW24-2 & 24 & $26.409-34.608$ & R1_77061 & 27.503 & 6.36 & 14.6 & GW \\
\hline & qBW24-3 & 24 & $46.786-50.373$ & R1_137885 & 47.525 & 4.76 & 10.6 & $C W$ \\
\hline & qBW24-4 & 24 & $53.208-53.942$ & R1_70232 & 53.435 & 5.31 & 11.6 & GW \\
\hline & qBW24-5 & 24 & $68.218-71.651$ & R1_175083 & 70.827 & 5.63 & 12.3 & GW \\
\hline & qBW24-6 & 24 & $73.194-74.727$ & R1_227285 & 73.194 & 4.49 & 13.4 & $C W$ \\
\hline \multirow[t]{3}{*}{$\mathrm{TL}$} & qTL2-1 & 2 & $36.998-38.312$ & R1_98424 & 37.607 & 6.5 & 16.4 & GW \\
\hline & qTL2-2 & 2 & $58.475-59.267$ & R1_192243 & 58.917 & 4.25 & 9.3 & $C W$ \\
\hline & qTL24-1 & 24 & $53.208-53.942$ & R1_70232 & 53.435 & 4.38 & 9.7 & $C W$ \\
\hline \multirow[t]{3}{*}{$B L$} & qBL2-1 & 2 & 30.983-35.899 & R1_91299 & 31.482 & 4.46 & 11.7 & $C W$ \\
\hline & qBL2-2 & 2 & $36.998-38.312$ & R1_98424 & 37.607 & 6.58 & 16.9 & GW \\
\hline & qBL2-3 & 2 & $58.402-59.267$ & R1_192243 & 58.917 & 4.26 & 9.3 & CW \\
\hline \multirow[t]{14}{*}{$\mathrm{BH}$} & $\mathrm{qBH} 2-1$ & 2 & $36.998-38.312$ & R1_98424 & 37.607 & 6.32 & 16.5 & GW \\
\hline & qBH5-1 & 5 & $5.054-9.952$ & R1_291555 & 8.877 & 4.41 & 9.8 & CW \\
\hline & qBH5-2 & 5 & $41.779-47.428$ & R1_129989 & 46.184 & 4.84 & 10.8 & $C W$ \\
\hline & $\mathrm{qBH} 7-1$ & 7 & $37.461-43.712$ & R1_85816 & 37.461 & 5.29 & 12.1 & $C W$ \\
\hline & $\mathrm{qBH} 7-2$ & 7 & $72.254-74.31$ & R1_61604 & 73.623 & 4.65 & 10.3 & $C W$ \\
\hline & $\mathrm{qBH} 7-3$ & 7 & $92.377-95.06$ & R1_92737 & 92.439 & 6.23 & 13.6 & GW \\
\hline & qBH8-1 & 8 & $32.209-33.716$ & R1_49419 & 33.402 & 4.21 & 9.7 & $C W$ \\
\hline & qBH9-1 & 9 & $42.675-44.714$ & R1_101372 & 44.120 & 4.53 & 9.9 & CW \\
\hline & qBH19-1 & 19 & $30.002-32.092$ & R1_140416 & 30.237 & 6.07 & 14.2 & GW \\
\hline & qBH19-2 & 19 & $63.418-69.448$ & R1_198192 & 66.225 & 4.76 & 10.8 & $C W$ \\
\hline & $\mathrm{qBH} 24-1$ & 24 & $14.585-19.843$ & R1_129529 & 17.156 & 5.61 & 12.3 & GW \\
\hline & $\mathrm{qBH} 24-2$ & 24 & $26.409-34.608$ & R1_77061 & 27.503 & 5.52 & 13.1 & GW \\
\hline & $\mathrm{qBH} 24-3$ & 24 & $53.208-53.942$ & R1_115171 & 53.435 & 5.54 & 12.2 & GW \\
\hline & $\mathrm{qBH} 24-4$ & 24 & $68.218-74.727$ & R1_175083 & 70.827 & 4.83 & 11.3 & $C W$ \\
\hline \multirow[t]{5}{*}{$\mathrm{CPH}$} & $\mathrm{qCPH} 2-1$ & 2 & $30.708-37.607$ & R1_98424 & 37.607 & 7.88 & 18.5 & GW \\
\hline & $\mathrm{qCPH} 2-2$ & 2 & $58.475-59.267$ & R1_192243 & 58.917 & 6.21 & 13.3 & GW \\
\hline & qCPH7-1 & 7 & $37.461-38.198$ & R1_85816 & 37.527 & 4.87 & 11.1 & CW \\
\hline & $\mathrm{qCPH} 7-2$ & 7 & $42.505-53.388$ & R1_235992 & 45.35 & 4.67 & 10.7 & CW \\
\hline & qCPH7-3 & 7 & $91.849-95.06$ & R1_93312 & 94.126 & 4.79 & 11 & $C W$ \\
\hline
\end{tabular}



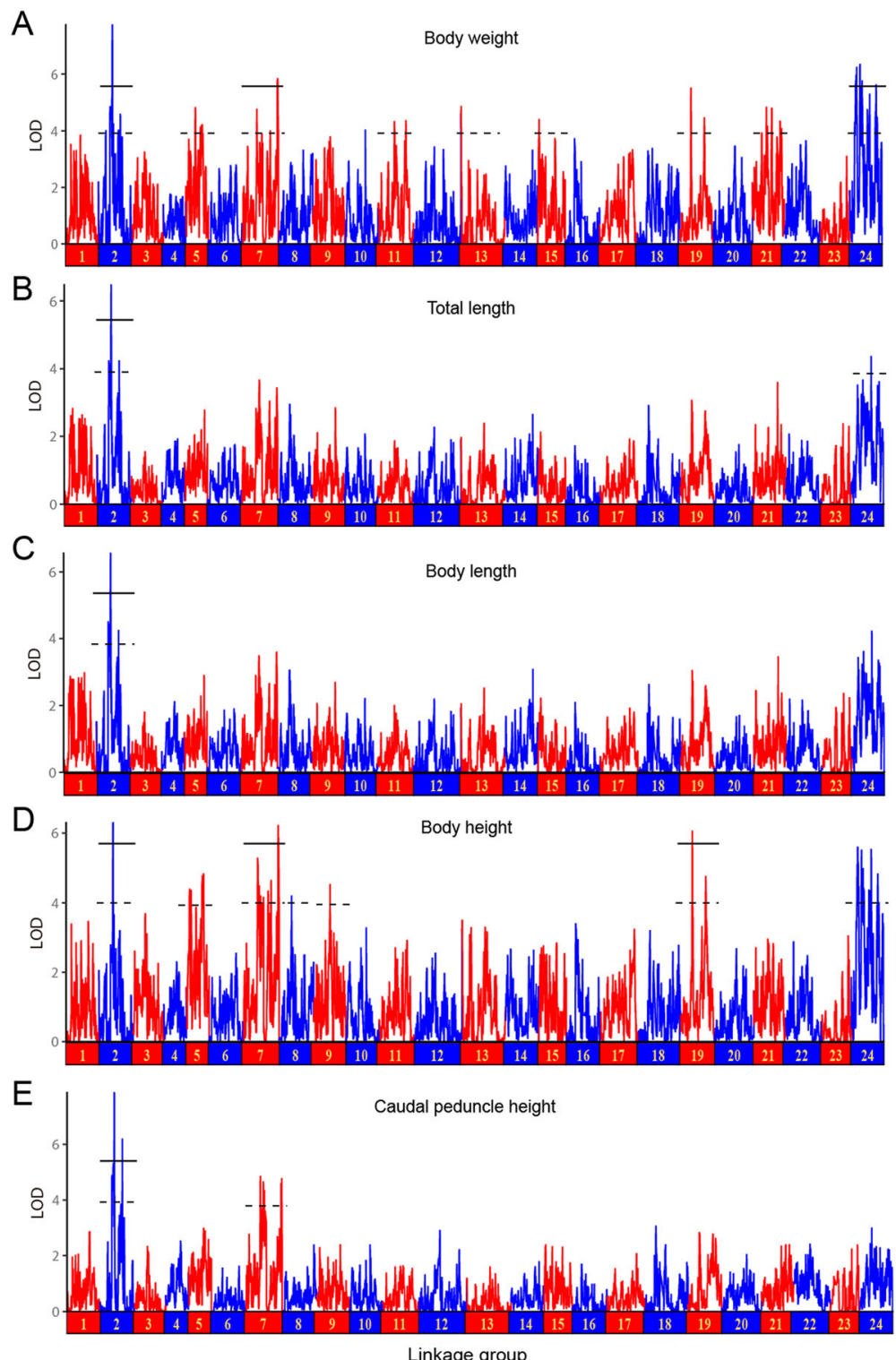

Fig. 3 LOD curves of growth-related QTL mapping for (a) body weight, (b) total length, (c) body length, (d) body height, and (e) caudal peduncle height in S.argus. The dashed and solid lines indicated the chromosome-wide and genome-wide significance thresholds, respectively. LOD significance threshold levels were determined on the basis of 1000 permutations at a significance level of 0.05 . The horizontal axes represent linkage groups LG1 - LG24

per linkage group, a shorter average marker interval, and longer ddRAD marker sequences. Meanwhile, there were improvements in density and resolution of S. argus map in comparison to maps constructed using RADseq methods in some other fishes, e.g., blunt snout bream Megalobrama amblycephala (237 SNPs per LG, $0.56 \mathrm{cM}$ ) [57], mandarin fish Siniperca chuatsi (137 SNPs per LG, 0.61 cM) [59], crucian carp (170 SNPs per LG, 0.44 cM) [65] and bighead carp Hypophthalmichthys nobilis (130 SNPs per LG, $0.75 \mathrm{cM}$ ) [66]. In Japanese flounder genetic map, although much more markers (12,712 SNPs) were assigned to 24 consensus LGs, a lower average marker interval $(0.47 \mathrm{cM})$ was found [16]. However, when compared to several ultra-high-density linkage maps consisting of tens of thousands markers, such as maps of $S$. salar [67] and Ictalurus punctatus [60, 68, 69], further improvements for the $S$. argus map are absolutely necessary. Nevertheless, as the first HD linkage map for S. argus and even for family Perciformes, this map not only can enable the fine-mapping of QTLs for important traits, but it also can provide useful tools for genetic and genomic studies in both $S$. argus and closely related species.

RAD markers are generally not randomly distributed across LGs, both marker-dense regions and some 


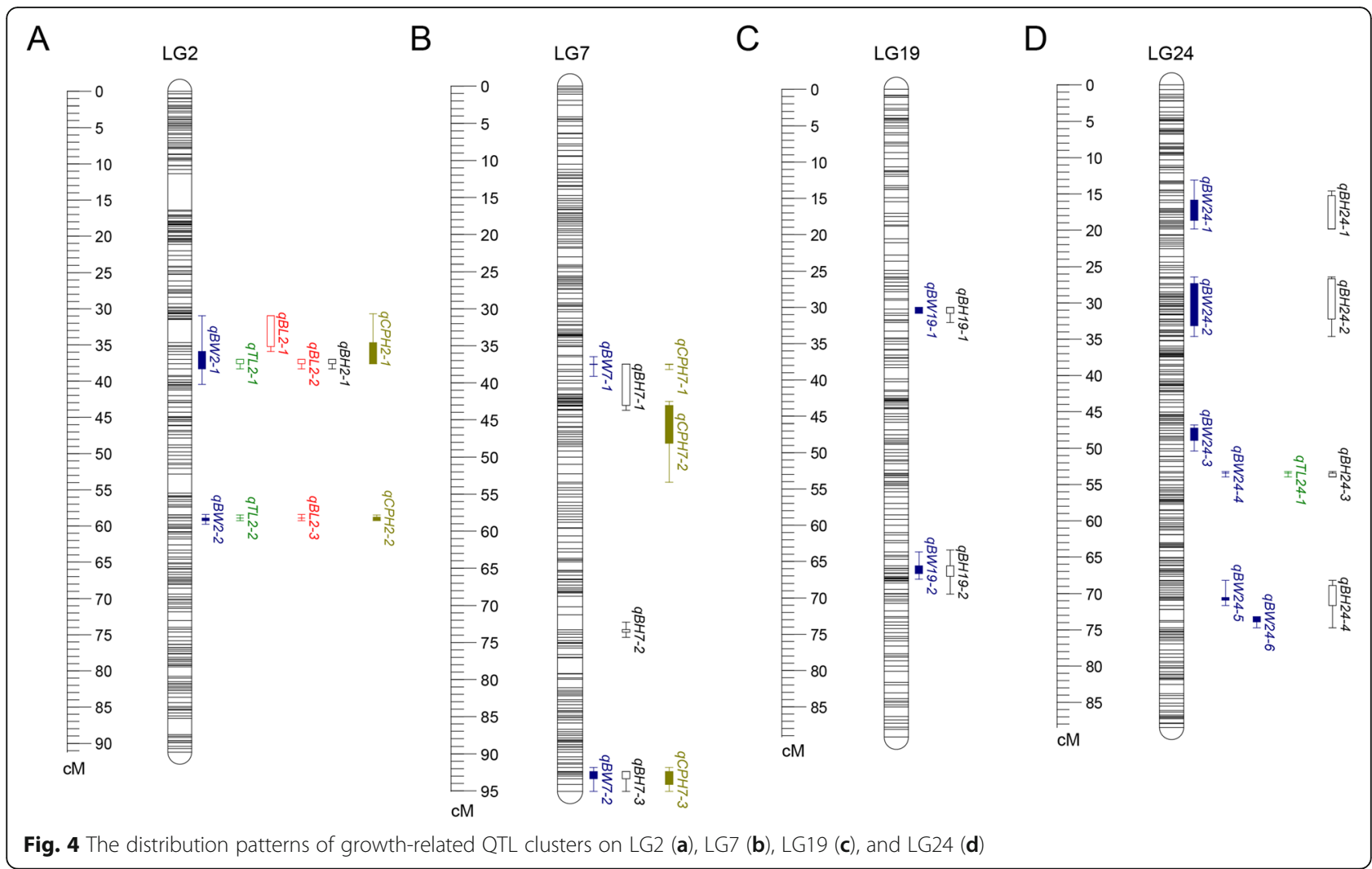

marker deserts can be clearly observed in linkage maps [70]. In our study, gaps shorter than $5.0 \mathrm{cM}$ accounted for an average of $98.0 \%$ of gaps. Although the average distance between adjacent markers was short $(0.35 \mathrm{cM})$, gaps greater than $5.0 \mathrm{cM}$ were not restricted to a particular group and were found on seven LGs (Table 4). This pattern may be one of the chief reasons for the non-random distribution of markers, as well as uneven marker polymorphism and recombination rates between female and male parents [12]. In the coming research, changing the types of restriction enzyme for RAD library construction or increasing the size of mapping population may be the alternative approaches to improve the $S$. argus genetic map.

\section{Comparative genome analysis}

Comparative mapping is an approbatory route for gene localization and function prediction, QTL identification, and genome evolution exploration in non-model fishes [9]. Here we analyzed the syntenic relationships between the $S$. argus genetic map and the genome of 10 teleosts. A relatively high-level of genomic synteny between $L$. crocea and S. argus were observed, about $15.0 \%$ of map markers had homologs in the $L$. crocea chromosomes (Fig. 2b). Most S. argus LGs and L. crocea chromosomes exhibited a high degree of syntenic relationship, there was only one $S$. argus LG (LG19) with a one-to-two relationship with L. crocea chromosomes (chr8 and chr16). Moreover, $13.0 \%$ of the homologous markers were located on various $L$. crocea chromosomes instead of conserved syntenic blocks, indicating a measure of intra-chromosomal rearrangements between $S$. argus and $L$. crocea. A similar phenomenon has been reported in the comparative analysis between southern catfish and channel catfish [71]. As a common chromosomal rearrangement mode in teleosts, translocation exists in many fishes. Translocation rates were believed to be positively correlated with lineage relationships between two fishes [72]. We also observed that each of two $O$. niloticus chromosomes was homologous with two $S$. argus LGs (chr7 with LG1 and LG16, chr21 with LG4 and LG9) (Fig. 2c). According to the hypothesis of previous studies, the ancestor of teleosts had the original chromosome number of 24, chromosomal fission and fusion played important parts in the formation of various chromosome numbers in fishes [73, 74]. Therefore, it could be inferred that two ancestral chromosomes of $O$. niloticus, which were homologous with LG1 and LG16, LG4 and LG9 of S. argus, respectively, fused and formed 22 chromosomes gradually during evolution.

\section{QTL mapping and candidate genes for growth traits}

A few preliminary studies on growth regulation have been reported in S. argus [75-77], but the principal 
Table 6 Prediction of candidate genes based on SNP markers associated with growth traits

\begin{tabular}{|c|c|c|c|c|c|}
\hline Marker & LG & Position (cM) & Scaffold & Annotation & Gene \\
\hline R1_192243 & LG2 & 58.917 & scaffold1197 & Protein shisa-6 & Shisa6 \\
\hline \multirow[t]{2}{*}{ R1_98424 } & \multirow[t]{2}{*}{ LG2 } & \multirow[t]{2}{*}{37.607} & \multirow[t]{2}{*}{ scaffold339 } & Cell death-inducing p53-target protein 1 & Cdip1 \\
\hline & & & & MKL/myocardin-like protein 2 & Mrtfb \\
\hline R1_235502 & LG5 & 42.973 & scaffold 1374 & Apoptotic protease-activating factor 1 & Apaf1 \\
\hline R1_269416 & LG5 & 25.992 & scaffold 1410 & Protein bicaudal D homolog 1-like & Bicd 1 \\
\hline R1_99132 & LG5 & 44.787 & scaffold195 & MANSC domain-containing protein 1 & Mansc1 \\
\hline R1_275284 & LG5 & 43.022 & scaffold273 & Probable tubulin polyglutamylase TTLL1 & $T t \| 1$ \\
\hline R1_151287 & LG5 & 40.377 & scaffold75 & Serine/threonine-protein kinase pim-3 & Pim3 \\
\hline R1_254692 & LG5 & 40.042 & scaffold86 & Nucleosome assembly protein 1-like 1 isoform X3 & Nap $1 / 1$ \\
\hline \multirow[t]{2}{*}{ R1_205729 } & \multirow[t]{2}{*}{ LG11 } & \multirow[t]{2}{*}{43.640} & \multirow[t]{2}{*}{ scaffold381 } & Fibronectin type-III domain-containing protein & Fndc \\
\hline & & & & LRRN4 C-terminal-like protein & Lrrn4Cl \\
\hline \multirow[t]{3}{*}{ R1_113228 } & \multirow[t]{3}{*}{ LG13 } & \multirow[t]{3}{*}{0.543} & \multirow[t]{3}{*}{ scaffold 1603} & Centrosomal protein of $131 \mathrm{kDa}$ isoform $\mathrm{X}_{1}$ & Cep131 \\
\hline & & & & E3 ubiquitin-protein ligase RNF213 & RNF213 \\
\hline & & & & Nuclear protein localization protein 4 homolog & Nploc4 \\
\hline R1_232824 & LG24 & 31.648 & scaffold 1004 & Cadherin-22-like isoform X3 & Cad221 \\
\hline \multirow[t]{2}{*}{ R1_119872 } & \multirow[t]{2}{*}{ LG24 } & \multirow[t]{2}{*}{31.488} & \multirow[t]{2}{*}{ scaffold1050 } & Transcription cofactor vestigial-like protein 4 & Vgll4 \\
\hline & & & & Ubiquitin-like modifier-activating enzyme ATG7 & Atg7 \\
\hline R1_216145 & LG24 & 70.541 & scaffold123 & Tribbles homolog 1 & Trib1 \\
\hline R1_269254 & LG24 & 17.017 & scaffold1587 & Protein FAM19A1 isoform $X_{1}$ & Fam19a1 \\
\hline R1_79166 & LG24 & 70.574 & scaffold1590 & LReO_3 protein & LReO_3 \\
\hline R1_287640 & LG24 & 70.906 & scaffold 1611 & ETS-related transcription factor Elf-3-like isoform X1 & Elf3l \\
\hline \multirow[t]{2}{*}{ R1_50011 } & \multirow[t]{2}{*}{ LG24 } & \multirow[t]{2}{*}{18.813} & \multirow[t]{2}{*}{ scaffold 1648} & Ataxin-1 & Atxn1 \\
\hline & & & & HMG box-containing protein 1-like & $H B P 1$ \\
\hline R1_255417 & LG24 & 18.653 & scaffold225 & Dynein heavy chain 1 , axonemal & Dnah17 \\
\hline \multirow[t]{3}{*}{ R1_115418 } & \multirow[t]{3}{*}{ LG24 } & \multirow[t]{3}{*}{31.488} & \multirow[t]{3}{*}{ scaffold551 } & Guanine nucleotide-binding protein G(s) subunit alpha & Galphas \\
\hline & & & & Leucine zipper protein 2 & Lzp2 \\
\hline & & & & Tubulin beta chain-like & Tubbl \\
\hline R1_137885 & LG24 & 47.525 & scaffold594 & Voltage-dependent calcium channel subunit alpha-2/delta-3 & Cacna2d3 \\
\hline R1_1854 & LG24 & 18.813 & scaffold619 & Prickle-like protein 2 & Prickle2 \\
\hline R1_219435 & LG24 & 31.194 & scaffold 820 & Phosphatidate cytidylyltransferase, mitochondrial & Tam41 \\
\hline R1_227285 & LG24 & 73.712 & scaffold839 & PDZ domain-containing protein 4 & $P d z d 2$ \\
\hline \multirow[t]{2}{*}{ R1_129529 } & \multirow[t]{2}{*}{ LG24 } & \multirow[t]{2}{*}{17.156} & \multirow[t]{2}{*}{ scaffold971 } & Protein kinase $\mathrm{C}$, delta & PKC $\Delta$ \\
\hline & & & & Scm-like with four MBT domains protein 1 & Sfmbt1 \\
\hline
\end{tabular}

LG Linkage group

components of growth traits QTL were still not investigated. Economically valuable traits of domestic animals are believed to be quantitative traits controlled by multiple loci or genes located in different genome regions [78]. In the present study, the HD linkage map enabled us to determine the number and position of the QTLs for growth traits. A number of significant QTLs associated with five growth traits were identified on different LGs (Table 5), indicating that growth-related traits are controlled by multiple QTL loci or regions in S. argus. Similar results were found in some other fish species and multiple QTLs were identified for one trait, e.g., 10 QTLs for body weight were detected on two LGs of $P$. ussuriensis [9], 14 QTLs for body height of T. blochii were were detected on two LGs [20], 4 QTLs for head length of common carp were detected on four LGs [3], and 5 significant QTLs for body length were detected on three LGs in Sander lucioperca [63]. These findings suggest that the growth traits are regulated by numerous loci or genes in fish, a few of these loci may have major effects and the remaining loci may possess minor effects. Moreover, the QTL regions were generally concentrated 
on certain groups (LG2, LG7 and LG24), suggesting that genes from different chromosomes may contribute to the same trait.

The phenotypes of five growth traits were found to be highly correlated with each other (Table 1). Interestingly, QTLs for these traits showed similar distribution patterns as expected and were mainly clustered into several main regions on S. argus genetic map. Many QTLs located in six clusters were associated with at least three growth traits, and several highly-overlapped QTL regions for BW and BH were detected on four LGs (Fig. 4 ), reflecting that these genomic regions may have key roles in growth regulation. Multifactorial QTLs for growth traits have also been detected in common carp [1, 3], L. calcarifer [18], S. chuatsi [59], S. lucioperca [63], bighead carp [66] and so on, implicating the pleiotropic effect of a QTL for multiple traits. Based on critical factors that control various traits through diverse metabolic pathways, pleiotropy may contribute to multifactorial QTLs [4]. It is generally believed that when closely-linked markers in multifactorial QTL regions were utilized in MAS, several correlated traits could be improved simultaneously. Thus, these QTL clusters will effectively facilitate the selective breeding of $S$. argus. However, our study is just the first attempt to locate growth-related QTLs, further verifications are needed to improve the scale and quality of identified QTLs.

A total of 32 candidate genes were identified in reference genome around the SNP markers located in the confidence intervals of QTLs related to body weight (Table 6). Among them, 15 genes are most likely to have some associations with growth, which indicated by the previous function studies in human or other mammalian species. Growth in most tissues is generally controlled by two different patterns: cell division and cell size increasing. $H b p 1$ is a transcription factor and a potent cell cycle inhibitor in normal and cancer cells, it activates or represses the expression of different cell cycle genes through direct DNA binding, cofactor recruitment, chromatin remodeling, or neutralization of other transcription factors [41]. Sfmbt1 acts as a key regulator affecting keratinocytes proliferation and apoptosis [42], and common deletions in this gene shows a significant association with fasting plasma glucose, implicating certain functions in some metabolic-related traits [43]. Vgll4 regulates muscle regeneration as a negative regulator of Yes-associated protein (YAP) in mice [44]. It is also a transcriptional cofactor acting as a novel tumor suppressor to inhibit cancer progression [45-47]. Current researches have revealed that Pim3, Cep131, Atxn1, and Nap1l1 play potential roles in tumorigenesis. Pim3 is correlated with enhanced tumor growth and cell survival [48] and its overexpression can enhance the proliferation of hepatoblastoma cells [49]. Cep131, a regulator for centrosome duplication and genome stability, can function as an oncogene and promote cell proliferation and migration in hepatocellular carcinoma [50]. Atxn1 has been demonstrated to be involved in tumorigenesis of cervical cancer cells via the EGFR-RAS-MAPK signaling pathway [51]. Whereas, Nap1l1 can promote the proliferation of induced pluripotent stem cells [52]. In addition, Prickle 2 and Cacna $2 d 3$ are two potential tumor suppressor genes in the development and progression of carcinoma [53, 54]. However, it should be aware of the fact that these genes are not necessarily the causative genes. Further studies are still needed to clarify the real associations between gene polymorphisms and growth traits in S.argus.

\section{Conclusions}

A high-density genetic linkage map with 6193 SNPs derived from ddRADseq data was constructed for $S$. argus with an average marker interval of $0.35 \mathrm{cM}$. Comparative mapping generates deeper insights into genomic evolution of $S$. argus by revealing a high level of syntenic relationship between this map and L. crocea genomes. A total of 44 significant QTLs for growth-related traits were identified on 11 LGs, and 19 of which were associated with body weight. Fifteen genes playing potential parts in cell proliferation and growth, tumorigenesis and muscle regeneration were recognized from some genome regions in QTL intervals. The S. argus genetic map will act as a useful tool in future genetic, genomic and evolutionary studies. These QTLs, SNP markers and candidate genes associated with growth traits lay the foundation for further genetic dissection of growth and can accelerate the marker-assisted breeding researches for genetic improvement in important traits of S. argus.

\section{Methods}

\section{Mapping family and phenotypic data}

In late May 2016, two wild $S$. argus populations were collected from Zhuhai offshore area $\left(21.50^{\circ} \mathrm{N}, 113.15^{\circ} \mathrm{E}\right.$; $N=46)$ and Taiwan Strait $\left(23.32^{\circ} \mathrm{N}, 120.60^{\circ} \mathrm{E} ; N=31\right)$. In August, a F1 full-sib family comprised of $\sim 8$ thousand larvae was produced by crossing of a dam from Taiwan Strait population and a sire from Zhuhai population. The larvae had been raised in a $5 \mathrm{~m} \times 5 \mathrm{~m} \times 2 \mathrm{~m}$ tank for 40 days. The juvenile offspring were then transferred and raised in a 0.3 ha disinfected muddy pond (water salinity was $15 \pm 5 \%$ ) at YuCheng Fish Fry Culture Farm in Zhuhai, Guangdong, China. The experimental fish were fed twice at 8:00 am and 6:00 pm each day. The fishery feed was purchased from YueHai Feeds Group Company in Guangdong, China. After 13 months of culture, a total of 420 progeny were randomly sampled for phenotypic measurements. Alive fish were anesthetized by immersing them in a tricaine methanesulfonate (MS-222) bath 
(300 mg/L), then phenotypic parameters of eight growth-related traits including body weight (BW), total length (TL), body length (BL), body height $(\mathrm{BH})$, predorsal length (PD), pre-anal length (PA), head length (HL) and caudal peduncle height $(\mathrm{CPH})$ were measured and recorded. The distribution patterns (normal or nonnormal) of phenotypic values of growth traits were determined by the Kolmogorov-Smirnov tests. To investigate the relationships among growth-related traits, Pearson's correlation coefficients $(r)$ were calculated. The statistical analyses were performed with the SPSS 19.0 software package (IBM, Armonk, NY, USA). Of the 420 progeny sampled, 200 individuals were randomly selected and subjected to genetic maps construction and growth-related QTL mapping. Fin clips of the two parents and full-sib progeny were excised and preserved in absolute ethanol at $-20^{\circ} \mathrm{C}$ until DNA isolation. Genomic DNA was extracted from fin clip tissues using a published traditional phenol-chloroform method with minor modification [79], its quality was evaluated using a Qubit fluorometer (Invitrogen, USA) and 1.0\% agarose gel electrophoresis. DNA samples with high purity $\left(\mathrm{A}_{260} / \mathrm{A}_{280}=1.8 \sim 2.0, \mathrm{~A}_{260} / \mathrm{A}_{230}=1.8 \sim 2.2\right)$ and good integrity (the primary band size $>20 \mathrm{~kb}$ ) will be utilized for the construction of ddRAD libraries. All DNA samples were adjusted to a concentration of $50 \mathrm{ng} / \mu \mathrm{L}$ by diluting with $10 \mathrm{mM}$ Tris-EDTA buffer. After sampling, the fish used in this study were still cultured in the muddy pond for further investigation.

\section{ddRAD library construction and sequencing}

In this study, a total of 202 genomic DNA samples from the two parents and their 200 offspring were used to construct ddRAD libraries by following the previously described protocol [11]. Briefly, $500 \mathrm{ng}$ of genomic DNA from each individual was double-digested with $20 \mathrm{U}$ of restriction enzymes EcoRI and NlaIII (New England Biolabs, Ipswich, MA, USA) in a $50 \mu \mathrm{L}$ reaction system at $37^{\circ} \mathrm{C}$ for $30 \mathrm{~min}$. The digested product was heatinactivated for $20 \mathrm{~min}$ at $65^{\circ} \mathrm{C}$, and then, was purified using a Qiagen MinElute Reaction Cleanup Kit (Qiagen, Valencia, CA, USA). The digested fragments were ligated with P1 [a unique 4-8 bp MID (multiplex identifier) for distinguishing each individual] and $\mathrm{P} 2$ adapters at $16^{\circ} \mathrm{C}$ over night. Whereafter, the ligated fragments were amplified ( 15 cycles of amplification) with High-Fidelity DNA Polymerase (Thermo Scientific, USA) using a set of primers that introduce sample specific barcode and sequencing primers. The PCR products were purified and size-selected (400-600 bp) via retrieval from an agarose gel. After insert-size analysis, the libraries were pooled with equal amount to prepare the final library that was sequenced in a lane of the Illumina HiSeq2500 platform (Illumina Inc., San Diego, CA, USA). In order to enhance sequencing coverage and improve genotyping accuracy, samples were merged in pools of $15 \sim 16$ samples for sequencing and genotyping the offspring, while the two parents were genotyped in a single pool, respectively. Overall, 15 pools ( 2 for the parents and 13 for the offspring) were sequenced with $150 \mathrm{bp}$ pair-end reads.

\section{Quality control and SNP genotyping}

Firstly, sequence reads for each individual were extracted and separated according to the specific MIDs using an in-house Perl script. The short reads without samplespecific MIDs and expected restriction enzyme motifs were discarded. Based on the quality score, the raw sequencing data were filtered with the Illumina read trimming tool Trimmomatic v0.39 [80] by removing adapters, the reads with bases quality below a threshold of 3 , and the reads with average Phred quality per base below 15. The resulting high-quality reads were then subjected to subsequent analyses. To identify loci in a set of individuals, the STACKS software (version 2.0 Beta 7; http://creskolab.uoregon.edu/stacks) [81] was utilized to de novo assemble the loci from the sequencing data for SNP calling. The libraries of loci were created by USTACKS, CSTACKS, SSTACKS and GENOTYPE, i.e., one for each individual and one for all the loci shared among the individuals. Minimum depth of coverage required to create a stack is 3; Maximum distance allowed between stacks is 3; 2 mismatches allowed between sample loci when build the catalog. Previously described parameters [59] were adopted in this study.

\section{Linkage map construction}

SNP loci that were heterozygous in at least one parent and genotyped in at least $90 \%$ of all the progeny were considered as high-quality markers and retained for further map construction. Based on their segregation patterns, SNP markers were categorized into three types: 1:1 (type $\operatorname{lm} \times$ ll or $n n \times n p$ ), 1:2:1 (type hk $\times$ hk) and 1:1:1:1 (type $\mathrm{ab} \times \mathrm{cd}$ or ef $\times \mathrm{eg}$ ). Linkage maps were constructed using JoinMap 4.1 [82] under the CP (cross pollinators) algorithm in this study. Chi-square tests were performed to examine the Mendelian segregation pattern of each SNP locus using the "Locus genot. Freq." function at the confidence level of 0.01. Markers with significant segregation distortion were excluded from linkage mapping analysis $(P<0.01)$. The linkage relationship between markers was examined by estimating logarithm of the odds (LOD) scores for recombination fraction. Linkage group assignments were made under a LOD threshold of 8.0. The regression mapping algorithm was selected for linkage mapping with the following settings: $\operatorname{Rec}=0.45$, LOD $=$ 1.0, Jump $=5$. Recombination frequencies were converted into map distances in centiMorgans (cM) using the 
Kosambi's mapping function. The estimated genome length $\left(G_{e}\right)$ was the average of lengths calculated by two previously described methods $[37,38]$. The resulting linkage maps were graphically visualized using MapChart 2.2 software [83].

\section{Comparative genomics}

ddRAD marker sequences (142-146 bp in length) were used as a query for homology searches against available fish genomes using BLASTn v.2.8.1+ (ftp://ftp.ncbi.nlm. nih.gov/blast/executables/blast+/LATEST) with an evalue cutoff of 1e-10. Reference sequences of Danio rerio (GRCz11), Oryzias latipes (ASM223469v1), Tetraodon nigroviridis (ASM18073v1), Gasterosteus aculeatus (ASM18067v1), O. niloticus (UMD1), Fugu rubripes (FUGU5), Ietalurus punetaus (IpCoco_1.2), Larimichthys crocea (ASM384579v1), P. olivaceus (ParOli_1.1), and S. salar (ICSASG_v2) were downloaded from the NCBI Genome Assembly database (ftp://ftp.ncbi.nlm.nih.gov/ genomes). Only ddRAD loci present in the linkage map and their putative homologs in the other fish genomes were taken into account in further analyses. For sequences that had multiple hits, only the ones with the lowest e-value were treated as their homologs and retained for comparative analysis. The final genomic synteny was visualized using the Circos software [84] release 0.69 (http://circos.ca/software/download/).

\section{QTL mapping for growth traits}

In this study the MapQTL 5.0 software [85] was employed to identify QTLs associated with growth traits following the mapping method of multiple QTL model (MQM). Regression algorithm was used for mapping quantitative trait loci in line crosses. Significant associations between loci and growth traits were detected with a mapping step size of $1.0 \mathrm{cM}$, and cofactors for MQM were automatically selected with a $p$-value of 0.05 . Both genome-wide and chromosome-wide significant LOD thresholds were estimated using 1000 permutation tests with a confident interval of $95 \%$. The location of each QTL was determined according to its LOD peak location and surrounding region. The percentage of the phenotypic variance explained by a QTL was estimated at the highest probability peak. Finally, the QTL profiles were visualized with MapChart 2.2 [83]. To identify candidate growth-related genes, ddRAD-tag sequences corresponding to SNP markers that were significantly correlated with growth traits were mapped on the $S$. argus reference genome (unpublished data) by BLASTn. Coding gene sequences were extracted from a $50 \mathrm{~kb}$ region containing $25 \mathrm{~kb}$ upstream and $25 \mathrm{~kb}$ downstream sequences flanking to each SNP locus. The gene functions were predicted subsequently based on the annotation information of genome. In addition, these gene sequences were also searched against the NCBI non-redundant $(\mathrm{Nr})$ protein database to verify their annotations using BLASTx with an e-value cut-off of $1.0 \times 10^{-10}$.

\section{Supplementary information}

Supplementary information accompanies this paper at https://doi.org/10. 1186/s12864-020-6658-1.

\begin{abstract}
Additional file 1 : Table S1. The statistics of eight growth-related traits of S. argus full-sib family. BW: body weight; TL: total length; BL: body length; $\mathrm{BH}$ : body height; PD: pre-dorsal length; PA: pre-anal length; HL: head length; $\mathrm{CPH}$ : caudal peduncle height; SD: standard deviation; $\mathrm{CV}$ : COefficient of variation.
\end{abstract}

Additional file 2 : Fig. S1. Frequency distribution of phenotypic values of eight growth-related traits of S. argus full-sib family. BW: body weight (g); TL: total length $(\mathrm{cm})$; $\mathrm{BL}$ : body length $(\mathrm{cm})$; $\mathrm{BH}$ : body height $(\mathrm{cm})$; PD: pre-dorsal length $(\mathrm{cm})$; PA: pre-anal length $(\mathrm{cm})$; $\mathrm{HL}$ : head length $(\mathrm{cm})$; $\mathrm{CPH}$ : caudal peduncle height $(\mathrm{cm})$

Additional file $\mathbf{3}$ : Table S2. Segregation patterns of heterozygotic SNP locus in the mapping population of S. argus.

Additional file 4 : Table S3. Statistic information of the sex-specific genetic maps of S. argus.

Additional file 5 : Fig. S2. Illustration of female linkage map for $\mathrm{S}$. argus. The map demonstrates the genetic lengths and marker distribution of 3512 SNP loci along the 24 linkage groups (LG1f-LG24f). The linkage groups are displayed by the vertical bars with black lines in each linkage group indicating a marker position. Genetic distance is shown by the vertical scale line with centiMorgans (cM).

Additional file $\mathbf{6}$ : Fig. S3. Illustration of male linkage map for S. argus. The map demonstrates the genetic lengths and marker distribution of 3627 SNP loci along the 24 linkage groups (LG1m-LG24m). The linkage groups are displayed by the vertical bars with black lines in each linkage group indicating a marker position. Genetic distance is shown by the vertical scale line with centiMorgans (cM).

Additional file 7 : Table S4. Estimation of the expected genetic distance of $\mathbf{S}$. argus linkage maps.

Additional file 8 : Fig. S4. Circos diagram representing the syntenic relationships between the consensus map and sex-specific maps of $S$. argus. (a) syntenic relationships between consensus map (right) and female map (left); (b) syntenic relationships between consensus map (right) and male map (left). Each colored arc represents a marker match between the linkage groups of two maps.

Additional file 9 : Table S5. QTL clusters for growth-related traits in S. argus.

\section{Abbreviations}

AFLP: Amplified fragment length polymorphism; GBS: Genotyping-bysequencing; LGs: Linkage groups; LOD: Logarithm of odds; MAS: Markerassisted selection; NGS: Next-generation sequencing; PVE: Phenotypic variance explained; QTL: Quantitative trait loci; RAD: Restriction siteassociated DNA; RAPD: Random amplified polymorphic DNA; SNP: Single nucleotide polymorphism; SSR: Simple sequence repeat

Acknowledgements

We thank YuCheng Fish Fry Culture Farm for supporting experimental fish culture.

Authors' contributions

$\mathrm{HC}, \mathrm{GL}$ and $W Y$ conceived the project; $W Y, C T$ and $\mathrm{HC}$ designed the experiments; WY, YW, HC and DJ performed the experiments; $C Z$ and $C T$ provided technical assistance; WY and YW performed data analysis; WY drafted the manuscript; and $G L$ and $C Z$ supervised and revised the manuscript. All authors read and approved the final manuscript. 


\section{Funding}

This work was supported by the National Key R\&D Program of China (2018YFD0901203), Special key projects of Guangdong science and Technology Department's science and technology innovation strategy (2018B030311050), National Natural Science Foundation of China (31702326, 41706174), Southern Marine Science and Engineering Guangdong Laboratory (Zhanjiang) (ZJW-2019-06), Natural Science Foundation of Guangdong Province (2019A1515010958, 2019A1515012042,

2018B030311050), Guangdong Ocean University Cultivating Fund Project for Excellent Doctoral Dissertations (201830), and Young Creative Talents Project of Guangdong Province Universities and Colleges (2017GkQNCX092). The funding bodies didn't include in the design of the study nor in collection, analysis, and interpretation of data, and also not in writing of the manuscript.

\section{Availability of data and materials}

The datasets supporting the findings of this article are included within the article and its supplementary information files. The raw sequencing data were deposited at the NCBI Sequence Read Archive (SRA) with the accession Number PRJNA578058.

\section{Ethics approval and consent to participate}

This study did not involve any endangered or protected species, hence no specific permissions and ethics were required to collect the fish samples. All experimental protocols involved in this study were approved by the Regulations for the Administration of Affairs Concerning Experimental Animals for the Science and Technology Bureau of China. All animal experiments were approved by the Animal Research and Ethics Committee of Guangdong Ocean University (NIH Pub. No. 85-23, revised 1996).

\section{Consent for publication}

Not applicable.

\section{Competing interests}

The authors declared no conflict of interest in the authorship and publication of this document.

\section{Author details}

'Southern Marine Science and Engineering Guangdong Laboratory (Zhanjiang), Guangdong Research Center on Reproductive Control and Breeding Technology of Indigenous Valuable Fish Species, Fisheries College, Guangdong Ocean University, Zhanjiang 524088, China. ${ }^{2}$ Food and Environmental Engineering Department, Yangjiang Polytechnic, Yangjiang 529566, China.

Received: 28 October 2019 Accepted: 9 March 2020

Published online: 03 April 2020

\section{References}

1. Wang X, Fu B, Yu X, Qu C, Zhang Q, Tong J. Fine mapping of growthrelated quantitative trait loci in Yellow River carp (Cyprinus carpio haematoperus). Aquaculture. 2018;484:277-85.

2. Yue GH. Recent advances of genome mapping and marker-assisted selection in aquaculture. Fish Fish. 2014;15(3):376-96.

3. Feng $X, Y u X$, Fu B, Wang $X$, Liu H, Pang M, et al. A high-resolution genetic linkage map and QTL fine mapping for growth-related traits and sex in the Yangtze River common carp (Cyprinus carpio haematopterus). BMC Genomics. 2018;19(1):230.

4. Song J, Li Q, Yu Y, Wan S, Han L, Du S. Mapping genetic loci for quantitative traits of golden shell color, mineral element contents, and growth-related traits in Pacific oyster (Crassostrea gigas). Mar Biotechnol. 2018:20(5):666-75.

5. Massault C, Bovenhuis H, Haley C, Koning D. QTL mapping designs for aquaculture. Aquaculture. 2008;285(1-4):23-9.

6. Young WP, Wheeler PA, Coryell VH, Keim P, Thorgaard GH. A detailed linkage map of rainbow trout produced using doubled haploids. Genetics. 1998;148:839-50.

7. Kocher TD, Lee WJ, Sobolewska H, Penman D, McAndrew B. A genetic linkage map of a cichlid fish, the tilapia (Oreochromis niloticus). Genetics. 1998;148(3):1225-32.
8. Slate J, Gratten J, Beraldi D, Stapley J, Hale M, Pemberton JM. Gene mapping in the wild with SNPs: guidelines and future directions. Genetica. 2009;136(1):97-107.

9. Zhu C, Liu H, Pan Z, Chang G, Wang H, Wu N, et al. Construction of a highdensity genetic linkage map and QTL mapping for growth traits in Pseudobagrus ussuriensis. Aquaculture. 2019;511:734213.

10. Davey JW, Hohenlohe PA, Etter PD, Boone JQ, Catchen JM, Blaxter ML, et al. Genome-wide genetic marker discovery and genotyping using nextgeneration sequencing. Nat Rev Genet. 2011;12:499-510.

11. Peterson BK, Weber JN, Kay EH, Fisher HS, Hoekstra HE. Double digest RADseq: an inexpensive method for de novo SNP discovery and genotyping in model and non-model species. PLoS One. 2012;7:e37135.

12. Sun X, Liu D, Zhang X, Li W, Liu H, Hong W, et al. SLAF-seq: an efficient method of large-scale de novo SNP discovery and genotyping using highthroughput sequencing. PLoS One. 2013;8:e58700.

13. Wang S, Meyer E, McKay JK, Matz MV. 2b-RAD: a simple and flexible method for genome-wide genotyping. Nat Methods. 2012;9:808-10.

14. Maroso F, Hermida M, Millan A, Blanco A, Saura M, Fernandez A, et al. Highly dense linkage maps from 31 full-sibling families of turbot (Scophthalmus maximus) provide insights into recombination patterns and chromosome rearrangements throughout a newly refined genome assembly. DNA Res. 2018;25(4):439-50.

15. Gonen S, Lowe NR, Cezard T, Gharbi K, Bishop SC, Houston RD. Linkage maps of the Atlantic salmon (Salmo salar) genome derived from RAD sequencing. BMC Genomics. 2014;15:166.

16. Shao C, Niu Y, Rastas P, Liu Y, Xie Z, Li H, et al. Genome-wide SNP identification for the construction of a high-resolution genetic map of Japanese flounder (Paralichthys olivaceus): applications to QTL mapping of Vibrio anguillarum disease resistance and comparative genomic analysis. DNA Res. 2015:22:161-70.

17. Palaiokostas C, Bekaert M, Khan MG, Taggart JB, Gharbi K, McAndrew BJ, et al. Mapping and validation of the major sex-determining region in Nile tilapia (Oreochromis niloticus L.) using RAD sequencing. PLoS One. 2013;8:e68389.

18. Wang L, Wan ZY, Bai B, Huang SQ, Chua E, Lee M, et al. Construction of a high-density linkage map and fine mapping of QTL for growth in Asian seabass. Sci Rep. 2015;5:16358.

19. Mackay TF, Stone EA, Ayroles JF. The genetics of quantitative traits: challenges and prospects. Nat Rev Genet. 2009;10:565-77.

20. Zhang G, Zhang X, Ye H, Jiang S, Yu H, Li J, et al. Construction of highdensity genetic linkage maps and QTL mapping in the golden pompano. Aquaculture. 2018:482:90-5.

21. Gupta S. An overview on morphology, biology, and culture of spotted scat Scatophagus argus (Linnaeus 1766). Rev Fish Sci Aquac. 2016;24(2):203-12.

22. Yang W, Chen H, Cui X, Zhang K, Jiang D, Deng S, et al. Sequencing, de novo assembly and characterization of the spotted scat Scatophagus argus (Linnaeus 1766) transcriptome for discovery of reproduction related genes and SSRs. J Oceanol Limnol. 2018:36(4):1329-41.

23. Barry TP, Castanos MT, Fast AW. Induced spermiation in the male spotted scat (Scatophagus argus) by long-term administration of 17amethyltestosterone followed by LHRHa. Asian Fish Sci. 1991;4(2):137-45.

24. Barry TP, Castanos MT, Macahilig MPSC, Fast AW. Gonadal maturation and spawning induction in female spotted scat (Scatophagus argus). J Aqua Trop. 1993:8:121-30

25. Cai ZP, Wang Y, Hu JW, Zhang JB, Lin YG. Reproductive biology of Scatophagus argus and artificial induction of spawning. J Trop Oceanogr. 2010;29(5):180-5.

26. Zhang MZ, Li GL, Zhu CH, Deng SP. Effects of fish oil on ovarian development in spotted scat (Scatophagus argus). Anim Reprod Sci. 2013; 141(1-2):90-7.

27. Li GL, Zhang MZ, Deng SP, Chen HP, Zhu CH. Effects of temperature and fish oil supplementation on ovarian development and fox/2 mRNA expression in spotted scat Scatophagus argus. J Fish Biol. 2015;86(1):248-60.

28. Gandhi V, Venkatesan V, Ramamoorthy N. Reproductive biology of the spotted scat Scatophagus argus (Linnaeus, 1766) from Mandapam waters, south-east coast of India. Indian J Fish. 2014;61(4):545-89.

29. Chen JH, He MX, Yan BL, Zhang JB, Jin SC, Liu L. Molecular characterization of dax1, and SF-1, and their expression analysis during sex reversal in spotted scat, Scatophagus argus. J World Aquac Soc. 2015;46(1):1-19.

30. Liu HF, Mu XJ, Gui LG, Su ML, Li H, Zhang G, et al. Characterization and gonadal expression of fox/2, relative to cyp19a genes in spotted scat Scatophagus argus. Gene. 2015;561(1):6-14. 
31. Chen HP, Deng SP, Dai ML, Zhu CH, Li GL. Molecular cloning, characterization, and expression profiles of androgen receptors in spotted scat (Scatophagus argus). Genet Mol Res. 2016;15(2):1-14.

32. Mustapha UF, Jiang DN, Liang ZH, Gu HT, Yang W, Chen HP, et al. Malespecific Dmrt1 is a candidate sex determination gene in spotted scat (Scatophagus argus). Aquaculture. 2018;495:351-8.

33. He FX, Jiang DN, Huang YQ, Mustapha UF, Yang W, Cui XF, et al. Comparative transcriptome analysis of male and female gonads reveals sexbiased genes in spotted scat (Scatophagus argus). Fish Physiol Biochem. 2019. https://doi.org/10.1007/s10695-019-00693-8.

34. Lan G, Yan B, Liao S, Luo Y, Xie R. Biology of spotted scat Scatophagus argus: a review. Fish Sci. 2005;24(7):39-41.

35. Cai Z, Hu J, Wang Y. Observing early development of Scatophagus argus. J Trop Oceanogr. 2014;33(4):20-5.

36. Suzuki A, Takeda M, Tanaka H, Yoo MS. Chromosomes of Scatophagus argus and Selenotoca multifasciata (Scatophagidae). Jpn J Ichthyol. 1988;35(1):1024.

37. Chakravarti A, Lasher LK, Reefer JE. A maximum likelihood method for estimating genome length using genetic linkage data. Genetics. 1991;128: 175-82.

38. Fishman L, Kelly AJ, Morgan E, Willis JH. A genetic map in the Mimulus guttatus species complex reveals transmission ratio distortion due to heterospecific interactions. Genetics. 2001;159:1701-16.

39. Said JI, Lin ZX, Zhang XL, Song MZ, Zhang JF. A comprehensive meta QTL analysis for fiber quality, yield, yield related and morphological traits, drought tolerance, and disease resistance in tetraploid cotton. BMC Genomics. 2013;14:776.

40. Li C, Dong YT, Zhao TL, Li L, Li C, Yu E, et al. Genome-wide SNP linkage mapping and QTL analysis for fiber quality and yield traits in the upland cotton recombinant inbred lines. Front Plant Sci. 2016;7:1356.

41. Bollaert E, Serra AD, Demoulin JB. The HMG box transcription factor HBP1: a cell cycle inhibitor at the crossroads of cancer signaling pathways. Cell Mol Life Sci. 2019;76(8):1529-39.

42. Li R, Qiao M, Zhao X, Yan J, Xi W, Sun Q. MiR-20a-3p regulates TGF-B1/ Survivin pathway to affect keratinocytes proliferation and apoptosis by targeting SFMBT1 in vitro. Cell Signal. 2018;49:95-104.

43. Chung RH, Chiu YF, Hung YJ, Lee WJ, Wu KD, Chen HL, et al. Genome-wide copy number variation analysis identified deletions in SFMBT1 associated with fasting plasma glucose in a Han Chinese population. BMC Genomics. 2017:18:591.

44. Feng $X$, Wang $Z Y$, Wang F, Lu TT, Xu JJ, Ma XY, et al. Dual function of VGLL4 in muscle regeneration. Embo J. 2019. https://doi.org/10.15252/embj. 2018101051.

45. Zhang WJ, Gao YJ, Li PX, Shi ZB, Guo T, Li F, et al. VGLL4 functions as a new tumor suppressor in lung cancer by negatively regulating the YAP-TEAD transcriptional complex. Cell Res. 2014;24(3):331-43.

46. Deng XC, Fang L. VGLL4 is a transcriptional cofactor acting as a novel tumor suppressor via interacting with TEADs. Am J Cancer Res. 2018;8(6): 932-43.

47. Zhang JM. VGLL4 suppresses the human breast cancer progression through the inhibition of YAP oncogenic function. Cancer Res. 2017. https://doi.org/ 10.1158/1538-7445.am2017-2581.

48. Wang GJ, Liu GH, Ye YW, Fu Y, Zhang XF. Bufothionine exerts anti-cancer activities in gastric cancer through Pim3. Life Sci. 2019. https://doi.org/10. 1016/j.lfs.2019.116615.

49. Stafman LL, Waldrop MG, Williams AP, Aye JM, Stewart JE, MroczekMusulman $\mathrm{E}$, et al. The presence of PIM3 increases hepatoblastoma tumorigenesis and tumor initiating cell phenotype and is associated with decreased patient survival. J Pediatr Surg. 2019;54(6):1206-13.

50. Liu XH, Yang YF, Fang HY, Wang XH, Zhang MF, Wu DC. CEP131 indicates poor prognosis and promotes cell proliferation and migration in hepatocellular carcinoma. Int J Biochem Cell B. 2017;90:1-8.

51. Kang AR, An HT, Ko J, Choi EJ, Kang S. Ataxin-1 is involved in tumorigenesis of cervical cancer cells via the EGFR-RAS-MAPK signaling pathway. Oncotarget. 2017;8(55):94606-18.

52. Yan $Y$, Yin PP, Gong H, Xue YY, Zhang GP, Fang B, et al. Nucleosome assembly protein 1-like 1 (Nap1/1) regulates the proliferation of murine induced pluripotent stem cells. Cell Physiol Biochem. 2016;38(1):340-50.

53. Rudenko EE, Gerashchenko GV, Lapska YV, Vozianov SO, Zgonnyk YM, Kashuba VI. PPM1M and PRICKLE2 are potential tumor suppressor genes in human clear-cell renal cell carcinoma. Biopolymers Cell. 2014;30(3):229-33.
54. Wong AMG, Kong KL, Chen LL, Liu M, Wong AMG, Zhu CL, et al. Characterization of CACNA2D3 as a putative tumor suppressor gene in the development and progression of nasopharyngeal carcinoma. Int J Cancer. 2013:133(10):2284-95.

55. Qiu GF, Xiong LW, Liu ZQ, Yan YL, Shen H. A first generation microsatellitebased linkage map of the Chinese mitten crab Eriocheir sinensis and its application in quantitative trait loci (QTL) detection. Aquaculture. 2016;451: 223-31.

56. Wang W, Hu Y, Ma Y, Xu L, Guan J, Kong J. High-density genetic linkage mapping in turbot (Scophthalmus maximus L.) based on SNP markers and major sex- and growth-related regions detection. PLoS One. 2015;10: e0120410.

57. Wan SM, Liu H, Zhao BW, Nie CH, Wang WM, Gao ZX. Construction of a high-density linkage map and fine mapping of QTLs for growth and gonad related traits in blunt snout bream. Sci Rep. 2017;7:46509.

58. Brown JK, Taggart JB, Bekaert M, Wehner S, Palaiokostas C, Setiawan AN et al. Mapping the sex determination locus in the hāpuku (Polyprion oxygeneios) using ddRAD sequencing. BMC Genomics. 2016;17:448.

59. Sun C, Niu Y, Ye X, Dong J, Hu W, Zeng Q, et al. Construction of a highdensity linkage map and mapping of sex determination and growth-related loci in the mandarin fish (Siniperca chuatsi). BMC Genomics. 2017;18:446.

60. Zhang S, Zhang X, Chen X, Xu T, Wang M, Qin Q, et al. Construction of a highdensity linkage map and QTL fine mapping for growth- and sex-related traits in channel catfish (Ictalurus punctatus). Front Genet. 2019;10:251.

61. Liu P, Wang L, Wong SM, Yue GH. Fine mapping QTL for resistance to VNN disease using a high-density linkage map in Asian seabass. Sci Rep. 2016;6: 32122.

62. Wang L, Chua E, Sun F, Wan ZY, Ye B, Pang H, et al. Mapping and validating QTL for fatty acid compositions and growth traits in Asian seabass. Mar Biotechnol. 2019;21(5):643-54.

63. Guo J, Li C, Teng T, Shen F, Chen Y, Wang Y, et al. Construction of the first high-density genetic linkage map of pikeperch (Sander lucioperca) using specific length amplified fragment (SLAF) sequencing and QTL analysis of growth-related traits. Aquaculture. 2018;497:299-305.

64. Hyten DL, Cannon SB, Song Q, Weeks N, Fickus EW, Shoemaker RC, et al. High-throughput SNP discovery through deep resequencing of a reduced representation library to anchor and orient scaffolds in the soybean whole genome sequence. BMC Genomics. 2010;11:38.

65. Liu H, Fu B, Pang M, Feng X, Yu X, Tong J. A high-density genetic linkage map and QTL fine mapping for body weight in crucian carp (Carassius auratus) using 2b-RAD sequencing. G3-Genes Genom Genet. 2017;7:2473-87.

66. Fu B, Liu H, Yu X, Tong J. A high-density genetic map and growth related QTL mapping in bighead carp (Hypophthalmichthys nobilis). Sci Rep. 2016;6: 28679.

67. Tsai HY, Robledo D, Lowe NR, Bekaert M, Taggart JB, Bron JE, et al. Construction and annotation of a high density snp linkage map of the Atlantic salmon Salmo salar genome. G3-Genes Genom Genet. 2016;6(7): 2173-9.

68. Li Y, Liu S, Qin Z, Waldbieser G, Wang R, Sun L, et al. Construction of a highdensity, high-resolution genetic map and its integration with BAC-based physical map in channel catfish. DNA Res. 2015;22(1):39-52.

69. Liu S, Li Y, Qin Z, Geng X, Bao L, Kaltenboeck L, et al. High-density interspecific genetic linkage mapping provides insights into genomic incompatibility between channel catfish and blue catfish. Anim Genet. 2016; 47(1):81-90.

70. Ji GS, Zhang QJ, Du RH, Lv P, Ma X, Fan S, et al. Construction of a highdensity genetic map using specific-locus amplified fragments in sorghum. BMC Genomics. 2017;18:51.

71. Xie M, Ming Y, Shao F, Jian J, Zhang Y, Peng Z. Restriction site-associated DNA sequencing for SNP discovery and high-density genetic map construction in southern catfish (Silurus meridionalis). Roy Soc Open Sci. 2018;5(5):172054.

72. Zhu C, Tong J, Yu X, Guo W. Comparative mapping for bighead carp (Aristichthys nobilis) against model and non-model fishes provides insights into the genomic evolution of cyprinids. Mol Gen Genomics. 2015;290:1313-26.

73. Kasahara M, Naruse K, Sasaki S, Nakatani Y, Qu W, Ahsan B, et al. The medaka draft genome and insights into vertebrate genome evolution. Nature. 2007:447:714-9.

74. Nakatani Y, Takeda H, Kohara Y, Morishita S. Reconstruction of the vertebrate ancestral genome reveals dynamic genome reorganization in early vertebrates. Genome Res. 2007;17:1254-65. 
75. Deng SP, Wu B, Zhu CH, Li GL. Molecular cloning and dimorphic expression of growth hormone $(\mathrm{gh})$ in female and male spotted scat Scatophagus argus. Fisheries Sci. 2014;80(4):715-23.

76. Li JT, Yang Z, Chen HP, Zhu CH, Deng SP, Li GL, et al. Molecular cloning, tissue distribution, and pharmacological characterization of melanocortin-4 receptor in spotted scat, Scatophagus argus. Gen Comp Endocr. 2016;230231:143-52

77. Deng SP, Chen HP, Zhai Y, Jia LY, Liu JY, Wang M, et al. Molecular cloning, characterization and expression analysis of spexin in spotted scat (Scatophagus argus). Gen Comp Endocr. 2018;266:60-6.

78. Goddard ME, Hayes BJ. Mapping genes for complex traits in domestic animals and their use in breeding programmes. Nat Rev Genet. 2009;10: 381-91.

79. Taggart JB, Hynes RA, Prodöuhl PA, Ferguson A. A simplified protocol for routine total DNA isolation from salmonid fishes. J Fish Biol. 1992:40:963-5.

80. Bolger AM, Lohse M, Usadel B. Trimmomatic: a flexible trimmer for Illumina sequence data. Bioinformatics. 2014;30:2114-20.

81. Catchen J, Hohenlohe PA, Bassham S, Amores A, Cresko WA. Stacks: an analysis tool set for population genomics. Mol Ecol. 2013;22:3124-40.

82. Van Ooijen JW. JoinMap 4, software for the calculation of genetic linkage maps in experimental populations. Netherlands: Kyazma BV, Wageningen; 2006.

83. Voorrips RE. MapChart: software for the graphical presentation of linkage maps and QTLS. J Hered. 2002;93:77-8.

84. Krzywinski M, Schein J, Birol I, Connors J, Gascoyne R, Horsman D, et al. Circos: an information aesthetic for comparative genomics. Genome Res. 2009;19:1639-45.

85. Van Ooijen JW. MapQTL 5.0, software for the mapping of quantitative trait loci in experimental populations. Wageningen: Academic; 2004. p. 1-57.

\section{Publisher's Note}

Springer Nature remains neutral with regard to jurisdictional claims in published maps and institutional affiliations.

Ready to submit your research? Choose BMC and benefit from:

- fast, convenient online submission

- thorough peer review by experienced researchers in your field

- rapid publication on acceptance

- support for research data, including large and complex data types

- gold Open Access which fosters wider collaboration and increased citations

- maximum visibility for your research: over $100 \mathrm{M}$ website views per year

At $\mathrm{BMC}$, research is always in progress.

Learn more biomedcentral.com/submissions 